\title{
Charge-Carrier Recombination in Halide Perovskites
}

Dane W. deQuilettes ${ }^{1,2}$, Kyle Frohna ${ }^{3}$, David Emin ${ }^{4}$, Thomas Kirchartz ${ }^{5}$, Vladimir Bulovic ${ }^{1}$, David S. Ginger ${ }^{2}$, Samuel D. Stranks ${ }^{3 *}$

${ }^{1}$ Research Laboratory of Electronics, Massachusetts Institute of Technology, 77 Massachusetts Avenue, Cambridge, Massachusetts 02139, USA

${ }^{2}$ Department of Chemistry, University of Washington, Box 351700, Seattle, WA 98195-1700, USA.

${ }^{3}$ Cavendish Laboratory, JJ Thomson Avenue, Cambridge CB3 0HE, United Kingdom

${ }^{4}$ Department of Physics and Astronomy, University of New Mexico, 1919 Lomas Blvd. NE, Albuquerque, New Mexico 87131, USA

${ }^{5}$ Faculty of Engineering and CENIDE, University of Duisburg-Essen, Carl-Benz-Str. 199, 47057

Duisburg, Germany

\section{Corresponding Author}

*sds65@cam.ac.uk 


\begin{abstract}
The success of halide perovskites in a host of optoelectronic applications is often attributed to their long photoexcited carrier lifetimes, which has led to charge-carrier recombination processes being described as unique compared to other semiconductors. Here, we integrate recent literature findings to provide a critical assessment of the factors we believe are most likely controlling recombination in the most widely studied halide perovskite systems. We focus on four mechanisms that have been proposed to affect measured charge carrier recombination lifetimes, namely: (1) recombination via trap states, (2) polaron formation, (3) the indirect nature of the bandgap (e.g. Rashba splitting), and (4) photon recycling. We scrutinize the evidence for each case and the implications of each process on carrier recombination dynamics. Although they have attracted considerable speculation, we conclude that shallow trap states, and the possible indirect nature of the bandgap (e.g. Rashba splitting), seem to be less likely given the combined evidence, at least in high-quality samples most relevant to solar cells and light-emitting diodes. On the other hand, photon recycling appears to play a clear role in increasing apparent lifetime for samples with high photoluminescence quantum yields. We conclude that polaron dynamics are intriguing and deserving of further study. We highlight potential interdependencies of these processes and suggest future experiments to better decouple their relative contributions. A more complete understanding of the recombination processes could allow us to rationally tailor the properties of these fascinating semiconductors and will aid the discovery of other materials exhibiting similarly exceptional optoelectronic properties.
\end{abstract}




\section{Biography}

Dane W. deQuilettes is a postdoctoral researcher in the Research Laboratory of Electronics at the Massachusetts Institute of Technology. He completed a dual-title $\mathrm{PhD}$ in Chemistry and Nanotechnology at the University of Washington as a National Science Foundation Graduate Research and Clean Energy Institute Fellow. He is the recipient of the Materials Research Society Graduate Student Gold Award, the 2018 IUPAC- Solvay International Young Chemist Award, and has recently been recognized as a Rising Star in the Natural Sciences by Nature.

Kyle Frohna is a PhD student in physics with Samuel D. Stranks in the Cavendish Laboratory at the University of Cambridge. He completed his undergraduate degree in Nanoscience and Materials Science at Trinity College, Dublin. He has worked previously with Professor Marco Bernardi at the California Institute of Technology on computing the transport properties of carriers in metal halide perovskites and with Professor Michael McGehee at Stanford University on the fabrication of high efficiency perovskite-silicon tandems.

David Emin received his Ph. D. in Physics from the University of Pittsburgh in 1968. After a postdoctoral position at UCLA, he joined the technical staff of Sandia National Laboratories in Albuquerque, New Mexico. He was elected a Fellow of the American Physical Society in 1977. In 1983 he was in the very small inaugural group promoted by Sandia National Laboratories to be a Distinguished Member of Technical Staff. He retired from Sandia but continues his research while an Adjunct Professor in the Department of Physics and Astronomy of the University of New Mexico. In 2008 David Emin was named an "outstanding referee" by the American Physical Society in its first round of selectees. 
Thomas Kirchartz received his $\mathrm{PhD}$ from the RWTH Aachen in 2009 and subsequently moved to Imperial College London working on organic solar cells with Prof. Jenny Nelson. In 2013, he was appointed Professor at the University of Duisburg-Essen (Electrical Engineering) and became head of a group working on characterization and simulation of solution-processable solar cells at the Research Centre Jülich. Since 2017, he is deputy head of the IEK-5 Photovoltaics, the institute dealing with all photovoltaics-related research at the Research Centre Jülich.

Vladimir Bulovic is the Fariborz Maseeh Chair in Emerging Technology and Director of the Massachusetts Institute of Technology's newest nanoscience and nanotechnology, MIT.nano. He received his M.S. Degree from Columbia University in 1993 and his Ph.D. from Princeton University in 1998. He is a recipient of the U.S. Presidential Early Career Award for Scientist and Engineers, the National Science Foundation Career Award, and the Bose Award for Distinguished Teaching.

David S. Ginger earned dual B.S. degrees in chemistry and physics from Indiana University in 1997 and a Ph.D. in physics from the University of Cambridge (U.K.) in 2001. After a postdoctoral fellowship at Northwestern University, he joined the University of Washington in 2003. He is currently Alvin L. and Verla R. Kwiram Endowed Professor of Chemistry and Associate Director of the University of Washington Clean Energy Institute.

Samuel D. Stranks is a Royal Society University Research Fellow at the Cavendish Laboratory and Lecturer in Energy in the Department of Chemical Engineering and Biotechnology at the 
University of Cambridge. He completed his $\mathrm{PhD}$ at the University of Oxford, investigating carbon nanotubes for organic solar cell applications. He then turned his focus to the optoelectronic properties of perovskites as a Junior Research Fellow at Oxford and then as a Marie Curie Fellow at MIT. He was awarded the 2018 Henry Moseley Medal and Prize from the Institute of Physics and the 2019 Marlow Award from the Royal Society of Chemistry for his contributions to the perovskite field. 
TOC GRAPHICS

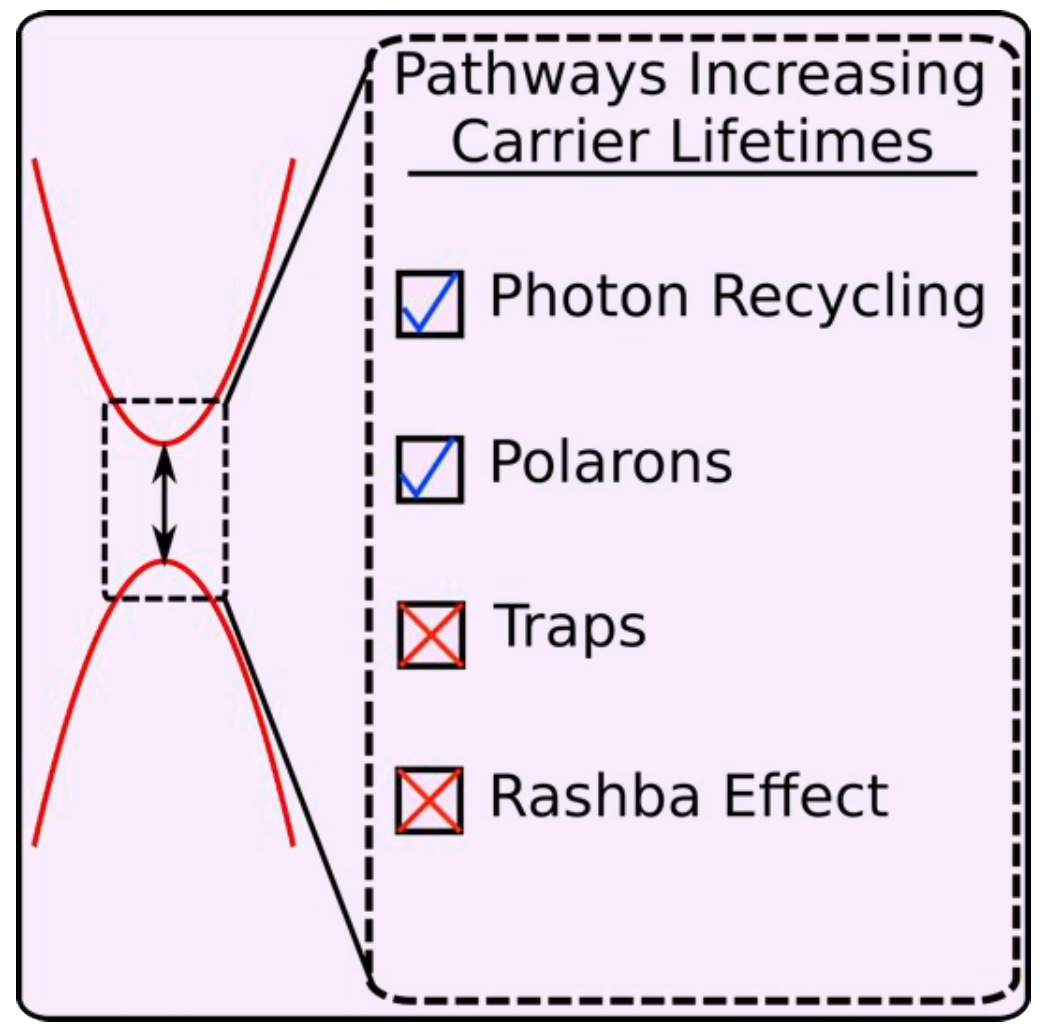

Table of Contents

Abstract ...................................................................................................................................2

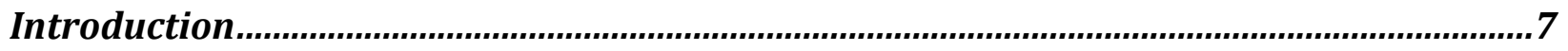

Trap-Related Recombination ( $\left.k_{1}\right)$ and Defect Tolerance ............................................ 10

Radiative Bimolecular Recombination (k2) and Optical Reciprocity .............................. 14

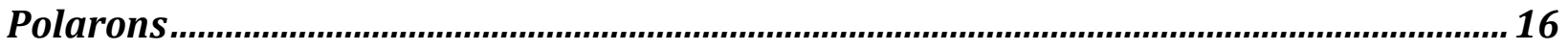

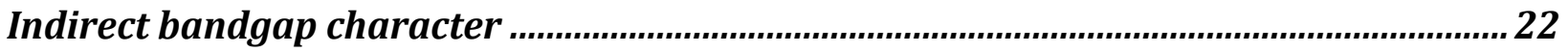

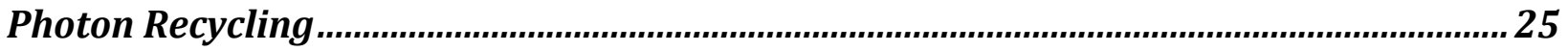

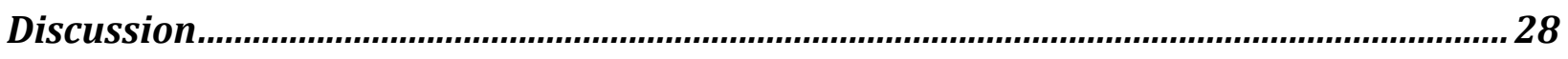

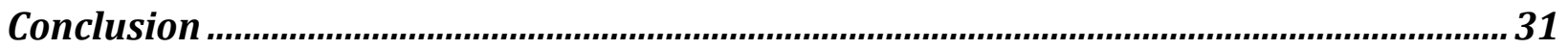




\section{Introduction}

The charge-carrier lifetime is an important metric in a photovoltaic device ${ }^{1}$, where longer carrier lifetimes have been shown to directly correlate with higher power conversion efficiency in solar cells such as GaAs. ${ }^{2}$ The recombination of charge carriers in a semiconductor can be summarized through the following simplified rate equation ${ }^{3,4}$ (see Supporting Information (SI) for assumptions and derivation):

$$
-\frac{d n}{d t}=k_{1} n+k_{2} n^{2}+k_{3} n^{3}
$$

where $n$ is the electron charge carrier density (Eqn. 1 assumes electron density equals hole density to facilitate qualitative discussion), $k_{1}$ is the first-order Shockley-Read-Hall (SRH) trapping (nonradiative) rate constant, $k_{2}$ is the second-order band-to-band (radiative) recombination rate constant, and $k_{3}$ is the third-order Auger (non-radiative) recombination rate constant. The photoexcited carrier density dictates which of the terms dominate recombination. Although lifetime is an important physical property of a semiconducting material, its usual definition as a characteristic time constant in an exponential decay only applies to a monomolecular decay process and therefore typically describes limiting cases of equation 1. For example, when recombination via traps dominates (in which the $k_{1}$ term dominates), or when the photoexcited carrier density is small compared to the majority carrier concentration (in which the $k_{2}$ term becomes pseudo-first order), non-radiative lifetime and minority carrier lifetime, respectively, are used to describe the dominant recombination pathway. For high-quality, intrinsic semiconductors, recombination kinetics no longer fall under these limiting cases and instead become a complex function dependent on the radiative recombination rate, the photoexcited charge carrier density, 
and interactions between these photoexcited carriers and defects (i.e. non-radiative recombination), the lattice, and each other (i.e. Coulomb interactions) $)^{5}$. The measured carrier decay rate ("lifetime") is therefore a combination of non-radiative and radiative recombination processes and in general does not follow monomolecular decay kinetics.

Metal halide perovskites, epitomized by the archetypical system methylammonium lead triiodide $\left(\mathrm{MAPbI}_{3}\right)$, are exciting materials for a variety of optoelectronic applications including solar photovoltaics ${ }^{6}$. In typical $\mathrm{MAPbI}_{3}$ films, the $k_{1}$ term dominates at photoexcitation densities $<10^{15}$ $\mathrm{cm}^{-3}, k_{2}$ dominates in the range $\sim 10^{15}-10^{17} \mathrm{~cm}^{-3}$, and $k_{3}$ dominates in the regime $>10^{17} \mathrm{~cm}^{-37,8}$. Charge recombination in perovskites has often been described as unique ${ }^{9-12}$ because even in a crudely solution-processed perovskite film with a seemingly high trap state density $\left(10^{14}-10^{17} \mathrm{~cm}^{-}\right.$ ${ }^{3}$ ), comparable to the photoexcited charge carrier density in an operating solar cell, the nonradiative lifetime $\left(1 / k_{1}\right)$ is long (>>100 ns) compared to typical direct gap semiconductors (of order nanoseconds). ${ }^{13}$ Indeed, photocarrier lifetimes in well-passivated perovskites have been reported to be as long as $10 \mu$ s under solar illumination fluences (i.e. low excitation fluences for most spectroscopic measurements) as measured by both photoluminescence and microwave conductivity. ${ }^{14,15}$ Such slow reported carrier decay rates are not expected from a material with strong absorption properties. Some reports have suggested that $k_{2}$ does not match what is theoretically predicted ${ }^{16}$ based on the principle of detailed balance, where strong absorption coefficients should lead to large radiative recombination rates. ${ }^{17-19}$ However, several reports have since shown that, when photon recycling is taken into account, ${ }^{5,20-23}$ the radiative bimolecular rate constant matches that which would be expected from reciprocity relations and is perhaps not 'slow' as previously thought. $5,15,20,24,25$ These results have established an on-going debate as to whether or not recombination in perovskites is truly unique. 
In this Focus Review, we examine the different recombination processes in the most widely reported metal halide perovskite systems. We focus on four common hypotheses that can influence the recombination terms associated with $k_{1}$ and $k_{2}$ in equation 1, each with varying levels of experimental and theoretical supporting evidence. These include long-lived trap states (Figure 1a), polaronic effects in which recombination is impeded via the interaction of the charge carriers with the lattice (Figure 1b), direct-indirect bandgap behavior induced from a Rashba effect (Figure 1c), and photon recycling (Figure 1d). We assemble the evidence supporting each of the four proposed mechanisms and, upon critical evaluation, determine that shallow trap states and the Rashba effect do not significantly impact carrier recombination. On the other hand, low transition rates into deep electronic trap states and photon recycling play a clear role in enabling long apparent lifetimes for high-quality samples. Furthermore, the impact of polaron formation on recombination dynamics deserves further study. We describe how many of these effects may inter-relate and in fact not be mutually exclusive, though likely also depend sensitively on the specific perovskite composition and structure. Finally, we identify routes to further decouple these effects and ultimately exploit this behavior. Understanding the recombination processes of these materials provides guidelines for designing similarly defect-tolerant semiconductors ${ }^{26}$ with favorable properties tailored towards specific applications. 

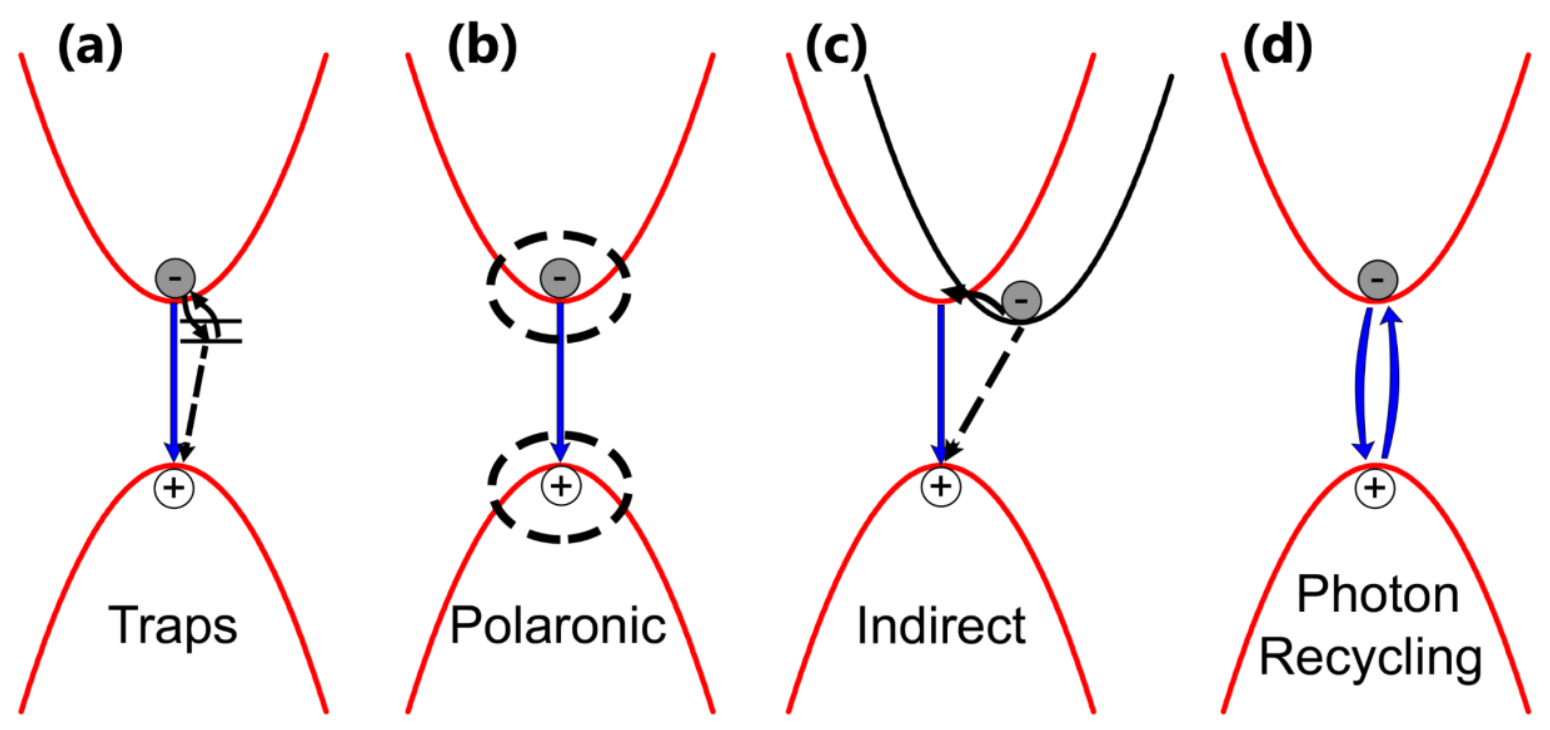

Figure 1. Proposed mechanisms that may impact charge carrier recombination in perovskites, namely (a) trapping, (b) polaronic effects, (c) indirect bandgap character, and (d) photon recycling.

\section{Trap-Related Recombination $\left(k_{1}\right)$ and Defect Tolerance}

Out of the four mechanisms we are highlighting in this perspective, the most well-studied to date is how sub-gap electronic states influence carrier recombination kinetics. ${ }^{27,28}$ Sub-gap states are expected to significantly influence the recombination kinetics at excitation powers relevant to photovoltaic operation where the steady-state carrier population $\left(\sim 10^{14}-10^{16} \mathrm{~cm}^{-3}\right)^{3,29}$ has been reported to be comparable to or below the sub-gap state population $\left(\sim 10^{14}-10^{17} \mathrm{~cm}^{-3}\right.$ for polycrystalline thin films). ${ }^{27,30,31}$ Indeed, several reports have now experimentally confirmed the presence of trap states within the band-gap of both polycrystalline and single crystal metal halide perovskites, ${ }^{32-35}$ and these trapped charges can exhibit micro- to milli-second lifetimes based on transient absorption and photoconductivity measurements. ${ }^{22,33}$

Importantly, the non-radiative lifetime is dependent on the rate at which carriers exchange energy with the lattice through phonon emission. This exchange of energy becomes generally less likely 
with an increasing ratio of energetic separation between the two electronic states compared to the energy of available phonons or vibrationally active modes. In inorganic semiconductors relevant for photovoltaic applications, direct band-to-band recombination via phonon emission is not favorable due to the high ratio of band gap energy to phonon energy. Reducing the energetic step of a single transition by introducing a localized state in the band gap will make phonon-mediated transitions substantially more likely. Thus, non-radiative recombination is typically described by a lifetime $(\tau)$ that is inversely proportional to the density of localized states $\left(N_{t}\right)$ in the band gap and a kinetic prefactor $(\Gamma)$ that describes the strength of electron-phonon coupling, and subsequently the likelihood of a transition via a single defect (i.e. $\tau \sim 1 / \Gamma N_{\mathrm{t}}$ ).

Recently, non-radiative rates for perovskites were calculated using well-known multiphonon emission theory, previously used for III-V semiconductors, ${ }^{36,37}$ which takes into account commonly reported optical phonon energies and electron-phonon coupling. ${ }^{38,39}$ The electronphonon coupling in halide perovskites is dominated by the inorganic metal-halide sublattice and, ${ }^{40}$ due to these heavy atomic components, the resulting phonon energies are characteristically lower than many semiconductors, making multiphonon processes less probable. ${ }^{39,41}$ Figure 2 shows the expected SRH non-radiative lifetimes for an optical phonon energy of $16.5 \mathrm{meV}^{42}$ for a given trap density and trap state energy within the bandgap $\left(E_{\mathrm{g}}=1.6 \mathrm{eV}\right)$, based on an extended version of the model previously reported (equations and calculations are reported in the SI). ${ }^{41}$ Optical phonons usually dominate these recombination processes in semiconductors as they produce the largest polarization field and allow for the largest changes in potential per unit displacement. ${ }^{43}$ Importantly, Figure 2 shows that for a typical trap density of $10^{15} \mathrm{~cm}^{-3}$ residing $0.6 \mathrm{eV}$ below (above) the conduction (valence) band, a non-radiative lifetime of $10 \mu \mathrm{s}\left(k_{1}=1 \times 10^{5} \mathrm{~s}^{-1}\right)$ is achievable. For comparison, high-quality samples of GaAs, which demonstrate 
photoluminescence quantum efficiencies in excess of 96\%, still exhibit non-radiative lifetimes of only tens of nanoseconds at $300 \mathrm{~K} .{ }^{13,44}$ This qualitative comparison shows that the trap-facilitated recombination for perovskites is characteristically slow. Figure 2 shows the functional form by which the perovskite nonradiative lifetime is sensitive to defects in the center of the gap and tolerant to shallow defects, as predicted by multiphonon theory and assuming no trap-trap transitions. As the low formation energy defects in perovskites are expected to reside near the band-edges, this may indeed render the lead-halide perovskite semiconductors tolerant to defects. ${ }^{45}$

We note that in addition to the multiphonon model, others have proposed that carrier lifetimes may be extended by multiple trapping and hopping processes in a band tail of shallow defect levels (within an energy of $\sim \mathrm{kT}$ from bandedges). ${ }^{32,46}$ These tail states could potentially be due to bandedge electronic energy fluctuations caused by the intrinsic polarizability of the $\mathrm{Pb}$ atom or the fast dipole reorientation of the methylammonium cation..$^{47,48}$ Nevertheless, due to the sharpness of the bandtail, it is likely that photo-excited carriers are rapidly re-emitted (de-trapped) from these trap states. In addition, the fact that surface passivation leads to enhancements in the photoluminescence quantum efficiency ${ }^{14,49,50}$ suggests that the trap states do not arise solely from static and/or dynamic disorder but rather likely point or extended defects at the surface and within the bulk of the perovskite. Finally, since both the mobility and, with it, the radiative bimolecular rate, go up with decreasing temperature, ${ }^{51,52}$ it seems unlikely that multiple trapping and reemission events are the dominant factor contributing to long carrier lifetimes. 


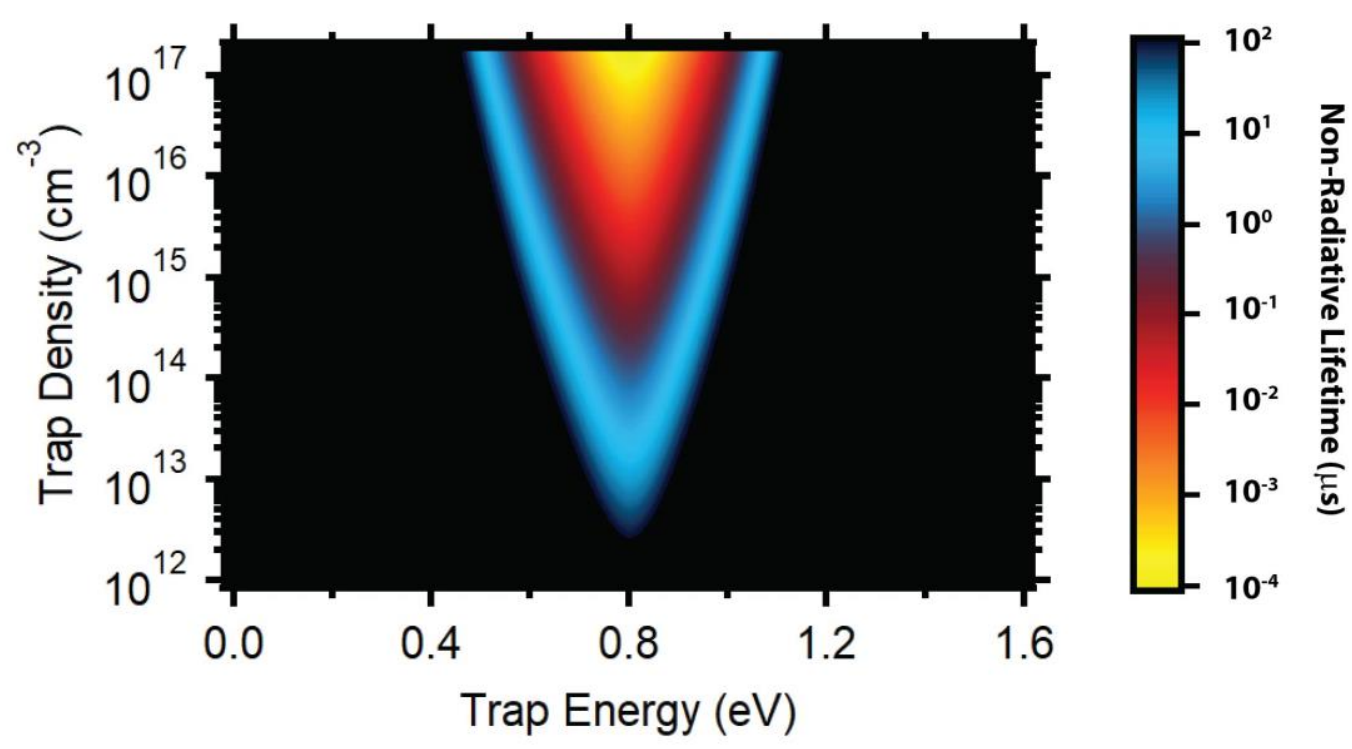

Figure 2. Calculated trap state densities leading to Shockley-Read-Hall (SRH) nonradiative lifetimes ranging from $0.1 \mathrm{~ns}$ to $100 \mu \mathrm{s}$. Here, trap energy is given relative to the conduction band $(0.0 \mathrm{eV})$ and the valence band resides at $1.6 \mathrm{eV}$ (i.e. bandgap of the material).

An accurate description of the role of these sub-gap electronic states in facilitating recombination still needs to be further developed. To date, trap states have primarily been probed electronically through deep level transient spectroscopy (DLTS), space-charge limited current (SCLC) analysis, and thermal admittance spectroscopy; these results have varied widely, ${ }^{27,53}$ likely due to variations in perovskite fabrication procedure as well as ion migration effects complicating the analyses. Interestingly, there are fewer studies that have shown optical signatures of trap states ${ }^{32,34}$, although some studies have predicted local trap densities by performing intensity-dependent PL measurements. ${ }^{29,54}$ These local variations in trap state densities over the sub-micrometer scale lead to spatial heterogeneity in charge carrier dynamics,${ }^{55-57}$ although the kinetics are also impacted by local composition and lattice distortions. ${ }^{58}$ The community would greatly benefit from further 
experimental evidence of the chemical nature and oxidation states ${ }^{59}$ of the subgap electronic states as well as resolving accurate energetic distributions and capture cross-sections. These studies would further inform theoretical studies, in which, thus far, calculated defect ionization levels have also widely varied depending on the level of theory used. ${ }^{60}$

\section{Radiative Bimolecular Recombination $\left(k_{2}\right)$ and Optical Reciprocity}

The rate of radiative recombination of a semiconductor is often described by the van RoosbroeckShockley (vRS) equation. Considering the principle of detailed balance, the radiative recombination rate for a given energy interval and energy is equal to the corresponding rate of generation of electron-hole pairs by radiation. ${ }^{18}$ The total recombination rate per unit volume per unit time $\left(R_{\mathrm{rad}}\right)$ is the energy-integrated recombination,

$$
R_{\mathrm{rad}}=\frac{8 \pi}{h^{3} c^{2}} \int_{0}^{\infty} \frac{E^{2} n_{r}^{2}(E) \alpha\left(E, f_{C}, f_{V}, T\right)}{e^{(E-\Delta \mu) / k_{B} T_{-1}}} d E\left(\mathrm{~cm}^{-3} \mathrm{~s}^{-1}\right)
$$

where $E$ is the (photon) energy, $h$ is Planck's constant, $c$ is the speed of light (in vacuum), $T$ is the temperature, $n_{r}(E)$ is the material's refractive index, $\Delta \mu$ is the quasi-Fermi level splitting, $k_{\mathrm{B}}$ is the Boltzmann constant, $\alpha\left(E, f_{C}, f_{V}, T\right)$ is the absorption coefficient of the bands under illumination, and $f_{\mathrm{c}}$ and $f_{\mathrm{v}}$ are the occupation probabilities for the conduction and valence bands (under illumination), respectively. We note that in this form of the vRS relation, $R_{\text {rad }}$ tends to zero as T approaches zero which is unphysical for a finite quasi-Fermi-Level splitting; a more rigorous treatment is therefore needed in some circumstances. ${ }^{61}$ Under thermal equilibrium, the radiative recombination rate $\left(R_{\text {rad, }, 0}\right)$ follows from eq. (2) by setting $\Delta \mu=0 .{ }^{18}$ The bimolecular recombination rate constant $\left(k_{2}, \mathrm{~cm}^{3} \mathrm{~s}^{-1}\right)$ as described in equation 1 is typically calculated using $k_{2}=R_{\mathrm{rad}, 0} / n_{\mathrm{i}}{ }^{2}$, where $n_{i}$ is the intrinsic carrier density. ${ }^{43}$ If we assume perovskite materials are intrinsic (i.e. Fermi- 
level lies in center of gap), $n_{\mathrm{i}}$ can be approximated using well-known theory for non-degenerate semiconductors based on the calculated density of states and measured bandgap of the material. ${ }^{62}$ Importantly, the rate extracted using this equation is the internal radiative recombination rate per unit volume and time, whereas an external recombination rate could be calculated knowing the properties of photon reabsorption (i.e. photon recycling) and escape for the geometry of the measured sample (see "Photon Recycling" section) ${ }^{63}$ Equation 2 has been used to accurately calculate the radiative recombination rate in $\mathrm{Si}^{64} \mathrm{Ge},{ }^{18} \mathrm{GaAs},{ }^{65}$ and $\mathrm{GaN},{ }^{66}$ although we note that there has been some debate on which absorption coefficient spectrum enters the equation, especially for materials with strong excitonic properties (see below). ${ }^{5}$

Following this theoretical framework, Figure 3a shows the calculated bimolecular rate constant from Davies et al. as a function of temperature. They show that when using Coulomb-enhanced absorption coefficients, in which the carriers are not well screened from one another and are bound electrostatically, they can reproduce the experimentally measured $k_{2}$ values (blue circles) reasonably well. These $k_{2}$ values are consistent with the internal recombination rates reported in the literature for halide perovskites $\left(0.7-6.8 \times 10^{-10} \mathrm{~cm}^{3} \mathrm{~s}^{-1}\right) \cdot{ }^{15,24,25}$ This figure also highlights that the room-temperature recombination rate is comparable to GaAs (i.e. $1.8-4 \times 10^{-10} \mathrm{~cm}^{3} \mathrm{~s}^{-1}$ ), ${ }^{67-69} \mathrm{a}$ high-performing, direct-bandgap photovoltaic material. Similar conclusions were recently attained through first-principles analyses. ${ }^{70}$ These results suggest that the fundamental band-to-band rate within the vRS approximation may be, in fact, close to what we might expect for the perovskite optical response and the radiative recombination would in fact not be considered as "slow" with respect to absorption. Nevertheless, reciprocity cannot distinguish between different effects modulating the measured bimolecular recombination rate constant (for example polarons, Rashba effect, and/or photon recycling) because any and all contributing radiative recombination 
mechanisms will already appear in the steady-state optical response. In other words, both emission and absorption processes are simultaneously occurring and are, therefore, both impacted by the presence of any of the mechanisms highlighted in this review. Indeed, this result merely reaffirms what we should expect, i.e. that optical reciprocity should always hold for a system in equilibrium. We now consider different recombination mechanisms that may influence the measured $k_{2}$ values.

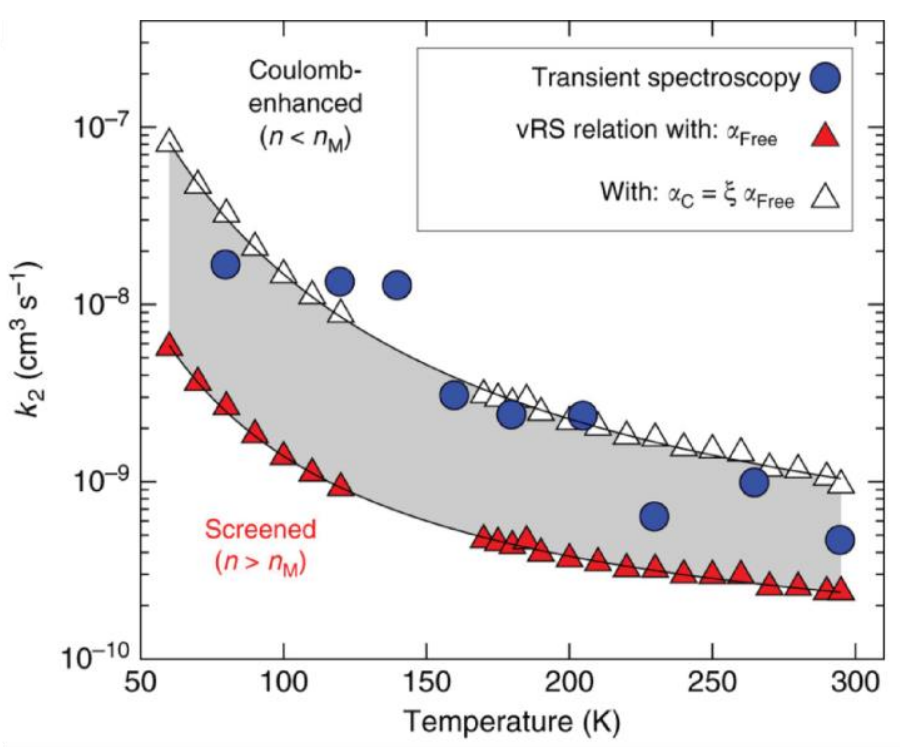

Figure 3. Bimolecular recombination rate constant $\left(k_{2}\right)$ as a function of temperature calculated using the van-Roosbroeck and Shockley (vRS) relation based on either the electron-hole continuum absorption coefficient including Coulomb interactions ( $\alpha_{C}$, white triangles), or the free (screened) electron-hole coefficient ( $\alpha_{\text {Free}}$, red triangles). Blue circles show values of intrinsic $k_{2}$ obtained previously $^{51}$ from transient terahertz photoconductivity spectroscopy. Reproduced with permission from Ref ${ }^{5}$. Copyright 2018 Springer Nature: Nature Communications under Creative Commons Attribution 4.0 International License, http://creativecommons.org/licenses/by/4.0/.

\section{Polarons}


In general, the energies of severely confined, localized electronic charge carriers depend strongly on the positions of the atoms that surround them. ${ }^{71}$ Nonetheless, displacements of the atoms surrounding electronic charge carriers can be ignored for commonly utilized high-mobility crystalline covalent semiconductors, such as $\mathrm{Si}$, where the electronic carriers move so fast that the surrounding atoms cannot respond to their presence. ${ }^{71-73}$ This assumption fails dramatically when electronic bands are sufficiently narrow or when electronic carriers are sufficiently confined by defects or slowed by disorder or, as discussed below, the lattice is highly polarizable. In particular, slow moving electronic charge carriers, even in well-ordered crystalline materials, will self-trap. ${ }^{71-}$ ${ }^{74}$ Then, an electronic carrier becomes bound within the potential well formed by displacements of surrounding atoms. A (strong-coupling) polaron refers to the composite unit comprising the selftrapped electronic charge carrier and the atomic-displacement pattern that produces its selftrapping. ${ }^{71,73}$ Polarons generally form and manifest distinctive optical, transport and recombination properties in ionic (polar) materials, and some groups have recently postulated that they may explain many of the extraordinary properties in metal halide perovskites. ${ }^{75,76}$ Indeed, since the 1950's charge carriers in even the simple perovskites, $\mathrm{SrTiO}_{3}$ and $\mathrm{BaTiO}_{3}$, have been recognized as polarons or bipolarons. ${ }^{77}$

Strong, short-range electron-phonon interactions, associated with the dependence of a charge carrier's energy on atoms it contacts, are omnipresent. However, additional long-range electronphonon interactions arise in ionic materials. In this case, Coulomb forces between electronic charge carriers and ions induce a long-range pattern of ionic displacements. The displaceability of ions is measured by the difference between the reciprocals of the relative dielectric constants at the high frequencies governing electronic polarization, $\varepsilon_{\infty}$, and that below which all atomic relaxation occurs, $\varepsilon_{0} \cdot{ }^{71,73}$ In typical ionic solids (e.g. alkali halides) in which polaron formation is 
prevalent, the static dielectric constant $\varepsilon_{0}(\sim 5)$ is almost twice the high-frequency dielectric constant. ${ }^{71}$ In halide perovskites, the much larger values of the quasi-static $\varepsilon_{0}(>30)$, along with the smaller values of the high frequency $\varepsilon_{\infty}(\sim 5-10)$, as measured by impedance spectroscopy, are consistent with a highly polarizable medium, which would be conducive to charge carriers forming polarons. ${ }^{75,78,79}$ Electrons in simple ionic solids, such as alkali halides, self-trap by displacing ions whereas electrons in simple polar liquids, such as water, self-trap by rotating surrounding polar molecules. Distinctively, the dielectric constant measurements on hybrid organic-inorganic lead perovskites find both ionic displacements and molecular rotations. ${ }^{75,80}$ The large-polaron binding energy given by Eq. (3) is proportional to $\left(1 / \varepsilon_{\infty}-1 / \varepsilon_{0}\right)^{2}$. The net large-polaron binding energy takes $\varepsilon_{0}$ to be the dielectric constant at frequencies below those of molecules' rotations and ions' vibrations ( MHz-GHz for perovskites), ${ }^{80}$ whereas the contribution from ions' displacements takes $\varepsilon_{0}$ to be the dielectric constant just below the characteristic optic-phonon frequency (i.e. $<1$ THz for perovskites).

A small polaron forms when its spatial extent collapses to that of a minimum-sized structural unit, $R_{\min }$, e.g. an atom, bond or fragment of a molecular solid. The long-range electron-phonon interactions of ionic materials also permit electronic charge carriers to form large polarons. ${ }^{71,81}$ In these cases, the spatial extent of a self-trapped electronic charge, its polaron radius $R_{\mathrm{p}}$, is no longer minimal. Formation of both types of polarons is facilitated in ionic solids where electronic carriers cannot out-run the effects of their long-range ionic displacements. Figure 4a depicts the scaling of the polaron energy $E$ with $R_{\mathrm{p}} / R_{\min }$ when the long-range and short-range electron-phonon interactions combine to produce a meta-stable small polaron and stable large polaron. ${ }^{71}$ The mobility of a large polaron generally exceeds $1 \mathrm{~cm}^{2} / \mathrm{Vs}$ and falls with increasing temperature, while the mobility of a small-polaron is usually $\ll 1 \mathrm{~cm}^{2} / \mathrm{Vs}$ and increases strongly with rising 
temperature ${ }^{60}$ Although some authors have predicted using density functional theory that charge carriers in halide perovskites form small polarons with large binding energies, ${ }^{82}$ the moderate mobilities ( 1-100 $\mathrm{cm}^{2} / \mathrm{Vs}$ at $\left.300 \mathrm{~K}\right)$, coherent transport, and mobility scaling as $T^{-\gamma}$, with $\gamma$ ranging from $0.5-1.7$, suggests large-polaron formation dominates. ${ }^{75,83}$ The net energy lowering with ions at their carrier-induced equilibrium positions is the large-polaron binding energy

$$
E_{p} \equiv-\frac{e^{2}}{4}\left(\frac{1}{\varepsilon_{\infty}}-\frac{1}{\varepsilon_{0}}\right) \frac{1}{R_{p}}
$$

where $R_{p} \equiv\left(\hbar^{2} / m e^{2}\right) /\left(\varepsilon_{\infty}^{-1}-\varepsilon_{0}^{-1}\right)$ denotes the polaron radius for a carrier with electronic effective mass $m$.

Interactions between polarons also generate distinctive effects. ${ }^{71,84,85}$ Recently, we predicted there will be a short-range repulsion between oppositely charged large polarons once they reach a critical distance close to the sum of the polaron radii. ${ }^{85}$ Figure $4 \mathrm{~b}$ further demonstrates this idea where, at short distances, the net large polaron energy increases primarily due to the overlap of the electronand hole-polaron atomic displacement patterns (black line), which overwhelms their Coulomb attraction (dotted line). In other words, there is a short-range repulsion that arises when two polarons of opposite charge approach one another, which may inhibit their coalescence and influence the radiative bimolecular recombination rate $\left(k_{2}\right)$ in perovskites. Specifically, shortrange repulsions between oppositely charged large polarons can reduce the bimolecular recombination rate below that for oppositely charged non-polaronic carriers diffusing with the mutual diffusion constant $D$ to within their Coulomb capture radius, $R_{\mathrm{c}} \equiv e^{2} / \varepsilon_{0} k T$. The bimolecular recombination rate from simple non-polaronic Coulomb capture is $k_{2}=4 \pi D R_{\mathrm{c}} \sim 10^{-7} \mathrm{~cm}^{3} / \mathrm{s}$, with representative parameters for metal-halide perovskites: $\mu=e D / k T=3 \mathrm{~cm}^{2} / \mathrm{Vs}$ and $\varepsilon_{0}=50$ at 300 K. ${ }^{86}$ The fact that smaller values of $k_{2}$ than this are actually measured $\left(k_{2} \sim 10^{-10} \mathrm{~cm}^{3} / \mathrm{s}\right)$ could be 
consistent with the short-range repulsion of oppositely charged large polarons influencing recombination. Furthermore, the asymmetry of the net polaron energy of Figure $4 \mathrm{~b}$ in the vicinity of its minimum indicates that the inter-polaron separation should increase as a function of temperature, leading to a reduction in the recombination rate with increasing temperature. Interestingly, this predicted behavior is consistent with previous reports of radiative recombination rates as a function of temperature but, ${ }^{87}$ we note, also consistent with the temperature dependence of $R_{\text {rad, } 0}$ (c.f. Eq. 2 and Fig. 4a). ${ }^{5}$ In addition, just as oppositely charged large polarons repel one another at short-range, the short-range attraction between like-signed large-polarons can drive their pairing as singlet large bipolarons; indeed the superconductivity of perovskite-oxide-based semiconductors has been attributed to their carriers forming large bipolarons. ${ }^{88-91} \mathrm{We}$ note that the present discussion focuses on the origin of the barrier to recombination of oppositely charged large polarons considering long-range Coulomb interactions. A treatment of excitons and their selftrapping requires consideration of the short-range interactions between excitons and the atoms they encompass and the interested reader is directed to Ref. ${ }^{92}$; such effects will become important in lower dimensional perovskite structures in which the exciton binding energy is significantly higher. 
(a)

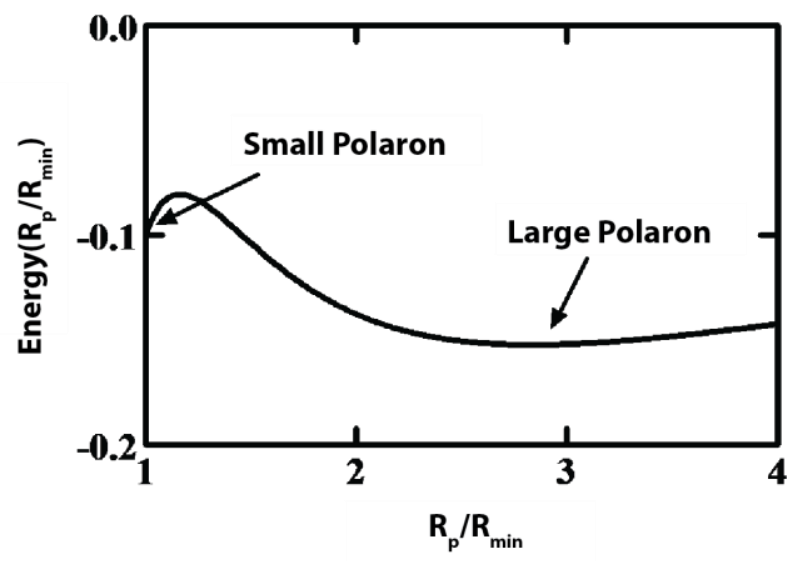

(b)

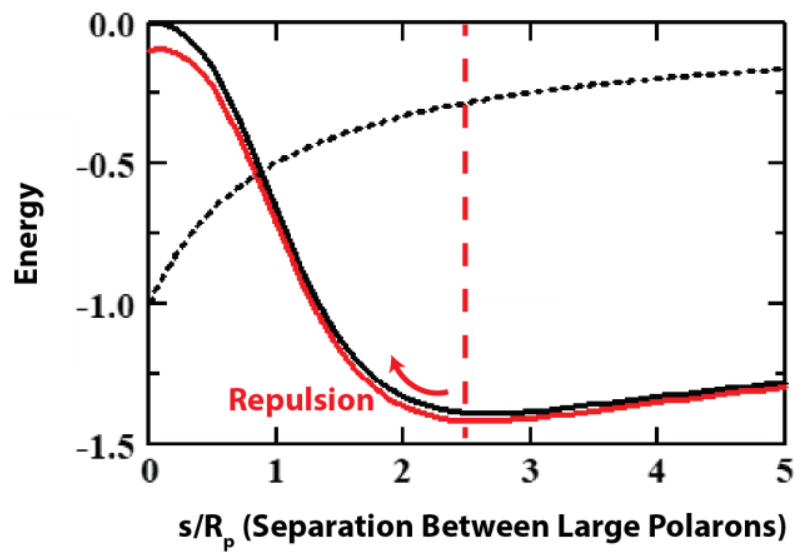

Figure 4. (a) The adiabatic polaron energy, the energy with the atoms fixed at their displaced equilibrium values, is plotted in arbitrary units versus the ratio of the large-polaron radius, $R_{\mathrm{p}}$, to the small polaron radius, $R_{\min }$. Here the large-polaron is stable with respect to the small-polaron. (b) The energy of two oppositely charged large polarons (solid black line) in units of their value at infinite separation plotted versus the separation of their centroids in units of their radii. Interference between the pattern of ionic displacements surrounding the two large polarons displaces the energy of their minimum to a value comparable to the sum of their radii (denoted by a red dashed line) ${ }^{85}$ For comparison, the dashed black line indicates the separation dependence of the Coulomb attraction between two oppositely charged polarons of finite size. The direct Coulomb attraction between the two polarons, inversely proportional to $\varepsilon_{0}$, makes only a relatively small contribution to perovskites' net interaction energy (red curve with $\left.\varepsilon_{0}=20 \varepsilon_{\infty}\right) .{ }^{82}$

Thus far, there are only a few studies presenting experimental evidence for polaron formation and its impact on recombination in halide perovskites. It has been proposed that polaron formation is dominated by the reorientation of the inorganic $\left(\mathrm{PbX}_{3}, \mathrm{X}=\mathrm{I}, \mathrm{Br}, \mathrm{Cl}\right)$ constituents ${ }^{93-95}$ and the 
dipolar cations may introduce dielectric drag, which negatively affects carrier mobility. ${ }^{75}$ Supporting this idea, Fleming and coworkers used 2D spectroscopy to show the slow dephasing time ( $10 \mathrm{ps})$ of a $0.9 \mathrm{THz}(3.7 \mathrm{meV})$ optical phonon signal suggesting strong coupling between the carriers and $\mathrm{Pb}-\mathrm{I}-\mathrm{Pb}$ angular distortions, but weaker interactions with other phonon modes, such as those involving the organic cation. ${ }^{40}$ The impact of polaron formation on hot carrier cooling rates has been explored in a few works, ${ }^{96,97}$ which qualitatively predict that polaron formation will also slow down the recombination of band-edge carriers. ${ }^{22,76,95}$ Recently, Asbury and coworkers have shown optical signatures of large polarons in the mid-IR decaying slower at elevated temperatures. ${ }^{87}$ They have attributed this to a reduction in the charge carrier mobility and a localization of polaron states at higher temperatures. Other studies have attempted to indirectly probe polaron recombination by investigating different perovskite compositions, $\mathrm{APbBr}_{3}$, with $\mathrm{A}^{+}$ $=\mathrm{CH}_{3} \mathrm{NH}_{3}{ }^{+}(\mathrm{MA}), \mathrm{NH}_{2} \mathrm{CHNH}_{2}{ }^{+}(\mathrm{FA})$, and $\mathrm{Cs}^{+}$, which all exhibit similar recombination kinetics. Interestingly, a similar experiment by Gong et al. showed a decrease in PL lifetime when methylammonium is deuterated, which they postulate to be due to the lower rotational frequency of this rotor affecting polaron formation. ${ }^{98}$ Future studies using direct optical probes of polaron formation and decay as a function of perovskite composition and structural phase will be critical in determining the impact of polaron formation on modulating $k_{2}$ recombination rates.

\section{Indirect bandgap character}

The Rashba effect has been proposed as a mechanism capable of retarding the radiative recombination pathway $\left(k_{2}\right)$ of carriers in perovskites through the creation of an indirect bandgap. ${ }^{99}$ In the presence of both spin-orbit coupling (SOC) and inversion symmetry breaking in the unit cell (Fig. 5a), carriers feel an effective magnetic field which splits the otherwise spin degenerate parabolic band edge in $k$-space. ${ }^{100}$ If the magnitude of splitting is different in the valence and 
conduction bands, an indirect bandgap is induced, the magnitude of which depends on the degree of symmetry breaking and spin orbit coupling. In the archetypal $\mathrm{MAPbI}_{3}$ composition, we recently showed that the room-temperature, tetragonal phase is centrosymmetric with space group I4/mcm, ${ }^{101}$ meaning that any observed Rashba effect could only be due to symmetry breaking at surfaces/grain boundaries or dynamic structural distortions. The direct experimental reports of the Rashba effect in metal-halide perovskites to date include the observation of a giant Rashba splitting in $\mathrm{MAPbBr}_{3}$ using angle-resolved photoelectron spectroscopy (ARPES), ${ }^{102}$ the measurement of circularly polarized photoluminescence in the presence of strong magnetic fields in isolated colloidal $\mathrm{CsPbBr}_{3}$ nanocrystals, ${ }^{103}$ and the observation of the circular photogalvanic effect in bulk $\mathrm{MAPbI}_{3}$ films. ${ }^{104}$ While these measurements do point to Rashba splitting, we note that the strongest evidence for Rashba splitting is derived from ARPES measurements (which is surfacesensitive) or measurements of quantum dot systems (which have high surface area to volume ratios).

We note that there are also conflicting reports in the field as to whether an indirect gap due to the Rashba effect would have a meaningful influence on the radiative recombination of carriers. Several groups have calculated $k_{2}$ in $\mathrm{MAPbI}_{3}$ from first-principles as a function of carrier density using unit cells relaxed in density functional theory. Azarhoosh et al. use a quasiparticle selfconsistent GW approximation to predict a considerable indirect gap due to a large difference in calculated splitting in the conduction band compared to the valence band. ${ }^{9}$ However, the calculated $k_{2}$ values of $\sim 10^{-12} \mathrm{~cm}^{3} \mathrm{~s}^{-1}$ at solar cell relevant carrier densities of $10^{15}-10^{17} \mathrm{~cm}^{-3}$ are considerably lower than values experimentally measured $\left(\sim 10^{-10} \mathrm{~cm}^{3} \mathrm{~s}^{-}\right)$. By contrast, Zhang et al. use the HSE hybrid functional in density functional theory (DFT) and find that there is a similar splitting in valence and conduction bands leading to calculated $k_{2}$ values in the range of $0.6-1.1 \times 10^{-10} \mathrm{~cm}^{3} \mathrm{~s}^{-}$ 
${ }^{1}$, depending on the degree of splitting. ${ }^{70}$ These results suggest that, to match experimental observations of radiative carrier recombination, the splitting must either be symmetric in the valence and conduction band or negligibly small.
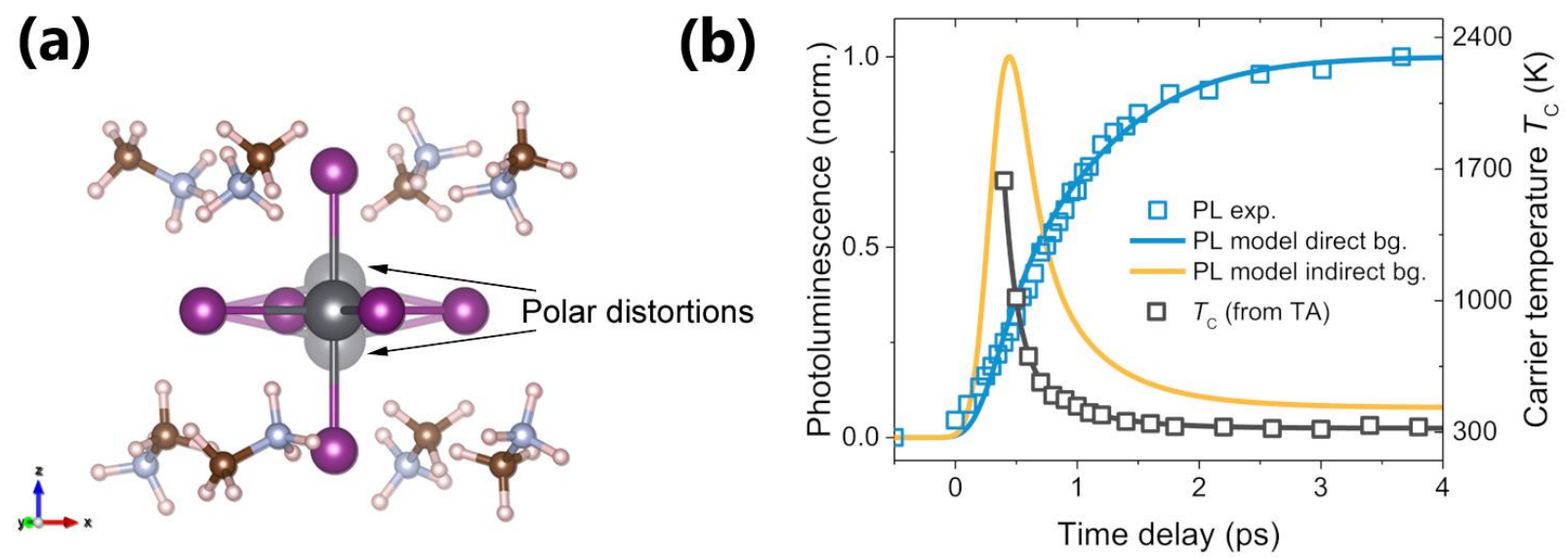

Figure 5. (a) Schematic of a single unit cell of $\mathrm{CH}_{3} \mathrm{NH}_{3} \mathrm{PbI}_{3}$ with distortions breaking inversion symmetry. (b) Ultrafast photoluminescence intensity (blue squares) and carrier population temperature (black squares), fitted with a Fermi-Dirac distribution state filling model for a direct gap (blue line) and an indirect gap (orange line). Adapted with permission from Wiley Publishing Group, ref. ${ }^{52}$, copyright 2018.

The influence of the Rashba effect on carrier recombination has been tested experimentally using both transient and steady-state measurements. ${ }^{103,105,106}$ Recent ultrafast transient grating PL measurements in both $\mathrm{MAPbBr}_{3}$ and $\mathrm{MAPbI}_{3}$ showed that photoexcited carrier population cooling could be modeled by a monotonic rise in PL intensity with decreasing carrier temperature (Figure 5b). ${ }^{52}$ This result is expected for a semiconductor with a direct bandgap as radiative recombination becomes more probable as carriers thermalizes to the band-edge (blue line). On the other hand, the PL intensity should decrease as a function of time for an indirect bandgap system as the carriers 
settle into the indirect gap with a different $k$-vector (orange line). A similar conclusion was reached by Sarritzu et al. who measured the "instantaneous" $\mathrm{PL}$ intensity of $\mathrm{MAPbI}_{3}$ and $\mathrm{MAPbBr}_{3}$ single crystals and films after a pulsed laser as a function of temperature. ${ }^{107}$ These results, along with the earlier theoretical arguments, are strong evidence that the Rashba effect does not play a significant role in impacting charge carrier recombination in typical 3D halide perovskite films being explored for many optoelectronics applications.

\section{Photon Recycling}

Photon recycling is a well-known phenomenon in high-quality, direct-gap semiconductors which exhibit high absorption coefficients, small Stokes-shift between absorption and emission spectra, efficient luminescence, and narrow emission escape cones ${ }^{25,108-110}$. This process directly impacts experimentally measured (external) $k_{2}$ values because emitted photons undergo multiple reabsorption/reemission events before eventually escaping from the film, leading to an apparent slowing of the recombination rate. ${ }^{111}$ Photon recycling is observed in doped GaAs, where it has been shown to enhance the photoluminescence lifetime to $139 \mathrm{~ns}$, which is beyond the theoretically calculated radiative lifetime of $13.5 \mathrm{~ns}$ at a doping density of $3.7 \times 10^{17} \mathrm{~cm}^{-3}$ (ref. ${ }^{68,112}$ ). As perovskite thin films also exhibit the prerequisite properties, ${ }^{113}$ photon recycling has also been demonstrated in these materials. ${ }^{108}$ The carrier decay rate equation (Eq. 1) can be extended to account for photon recycling by multiplying the internal recombination rate by the escape probability $\left(P_{\text {esc }}\right)$ to yield the external recombination rate (i.e. $\left.k_{2}^{\text {ext }}=P_{\text {esc }} k_{2}^{\text {int }}\right)^{108}$ (see SI for a full description, parameter values and modelling), 


$$
-\frac{d n}{d t}=k_{1} n+P_{e s c} k_{2}^{i n t} n^{2}+k_{3} n^{3}
$$

For example, Herz and coworkers have reported around an order of magnitude reduction in $k_{2}^{\text {ext }}$ as compared to $k_{2}^{\text {int }}$ due to the low escape probability $(\sim 5 \%)$ resulting from the high refractive index ( 2.5) of metal halide perovskites. ${ }^{24}$

(a)

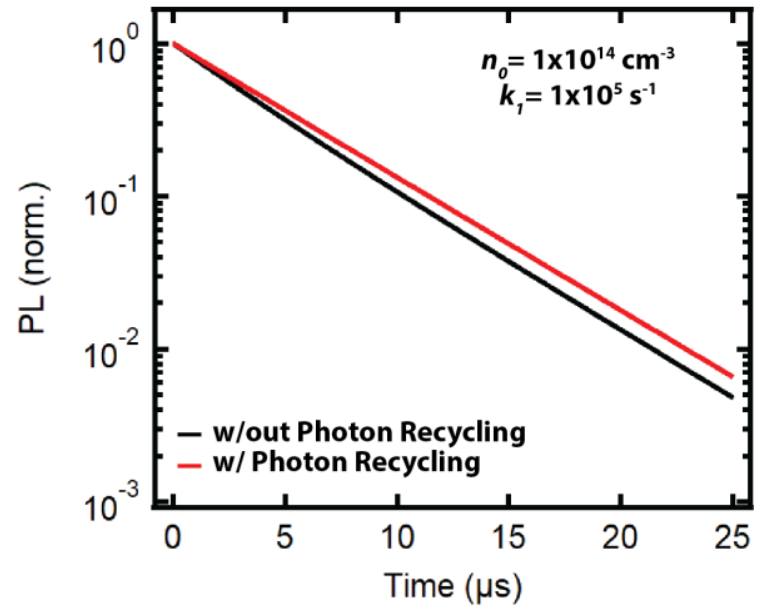

(c)

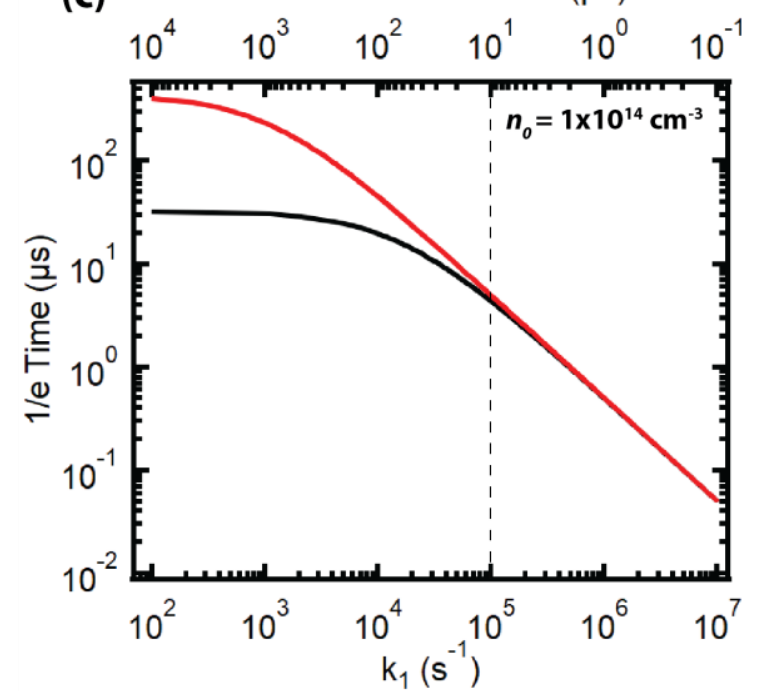

(b)

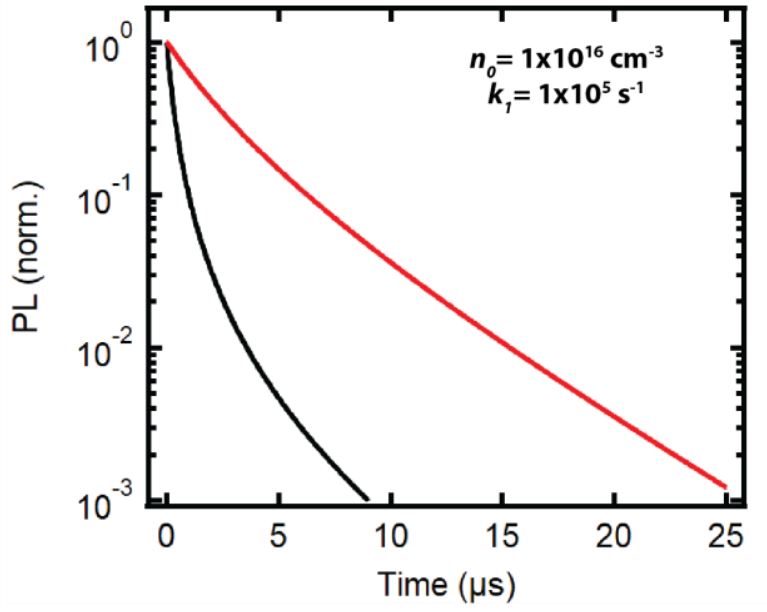

\begin{tabular}{llllll} 
(d) & \multicolumn{6}{c}{ Non-Radiative Lifetime ( $\mu \mathrm{s})$} \\
$10^{4}$ & $10^{3}$ & $10^{2}$ & $10^{1}$ & $10^{0}$ & $10^{-1}$
\end{tabular}

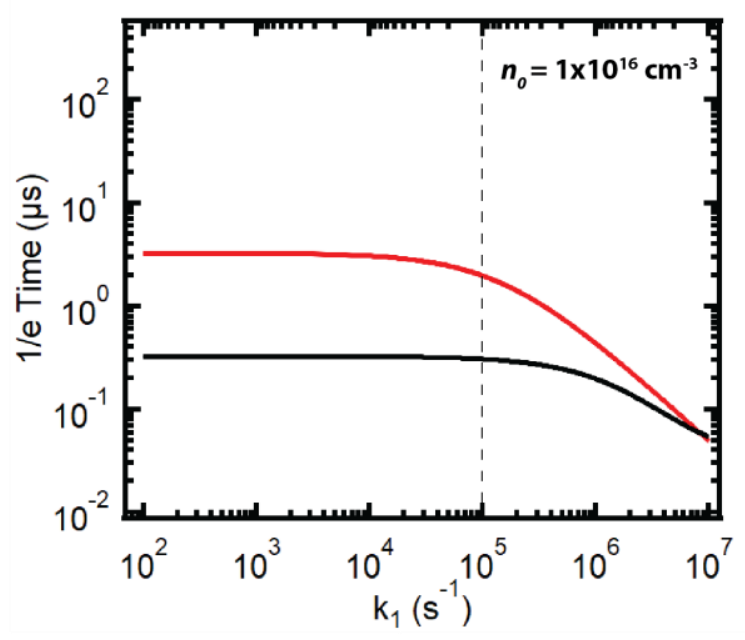

Figure 6. Simulated time-resolved photoluminescence (PL) with and without photon recycling at initial carrier densities of (a) $n_{0}=10^{14} \mathrm{~cm}^{-3}$, (b) $n_{0}=10^{16} \mathrm{~cm}^{-3}$ with $k_{1}=10^{5} \mathrm{~s}^{-1}, k_{2}=2 \times 10^{-10} \mathrm{~cm}^{3} \mathrm{~s}^{-1}$, and an escape probability, $P_{\mathrm{esc}}=7.4 \%$. (c,d) Time it takes for the PL intensity to fall to 1/e $(\sim 0.37)$ 
of the initial PL intensity $\left(\mathrm{PL}_{t=0}\right)$ as a function of $k_{l}$. Vertical dashed lines are the $k_{l}$ values $\left(10^{5} \mathrm{~s}\right.$ $\left.{ }^{1}\right)$ that correspond to the decay traces reported in (a) and (b).

Figures 6a and b show simulated time-resolved photoluminescence with (red) and without (black) photon recycling at initial carrier densities $\left(n_{0}\right)$ of $10^{14} \mathrm{~cm}^{-3}$ and $10^{16} \mathrm{~cm}^{-3}$, which span typical initial conditions for many time-resolved PL setups and close to the calculated steady-state carrier densities in solar cells. In these simulations, $k_{1}=10^{5} \mathrm{~s}^{-1}$ (typical reported values in high-quality $\mathrm{MAPbI}_{3}$ thin films) ${ }^{14,15}$ and photon recycling only has a sizeable impact on the decay kinetics at higher initial carrier densities (Figure $6 \mathrm{~b}$ and $\mathrm{d}$ ) at which radiative bimolecular recombination dominates over the first-order non-radiative recombination rate. To qualitatively show the trends in the decay kinetics for different values of $k_{l}$, Figure $6 \mathrm{c}$ and $\mathrm{d}$ show the $1 / \mathrm{e}$ times (time it takes for the PL to fall to $1 / \mathrm{e}$ of $\left.\mathrm{PL}_{\mathrm{t}=0}\right)$ for the two initial carrier densities. At low carrier densities $\left(n_{0}=\right.$ $1 \times 10^{14} \mathrm{~cm}^{-3}$, Figure $6 \mathrm{c}$ ), photon recycling does not have an influence on the decay time until $k_{1}$ drops below $10^{5} \mathrm{~s}^{-1}$, whereas photon recycling effects are clearly evident at $\sim 6 \times 10^{6} \mathrm{~s}^{-1}$ for initial carrier densities of $10^{16} \mathrm{~cm}^{-3}$ (Figure 6d). At low $k_{1}$ values the simulated data eventually plateau, at which point recombination is limited only by the bimolecular recombination rate rather than non-radiative recombination. Importantly, these data show that photon recycling is not expected to have a significant role in extending the carrier lifetime in perovskite thin films unless the trapping rate is low and/or a high carrier density can be maintained; both of these naturally infer a high internal PLQE (> 50\%, external PLQE of typical halide perovskite films $>10 \%)^{114}$. These results are broadly consistent with our recent studies showing that photovoltaic performance benefits from photon recycling when $k_{1}$ values and external electroluminescence efficiencies are 
$<5 \times 10^{5} \mathrm{~s}^{-1}$ and $>10 \%$, respectively. ${ }^{115}$ Thus, this phenomenon will be prominent in wellpassivated, high-quality perovskite optoelectronic devices.

\section{Discussion}

The multiphonon non-radiative and van-Roosbroeck Shockley radiative recombination rate calculations are both classical theoretical approaches to evaluate $k_{l}$ and $k_{2}$, respectively. Although these values serve as good reference points to evaluate experimentally extracted rate constants, we highlight that even within the same theoretical framework, several authors have reported $k_{2}$ values ranging from $\sim 10^{-10}-10^{-9} \mathrm{~cm}^{3} \mathrm{~s}^{-1}$. If the values are indeed $\sim 10^{-9} \mathrm{~cm}^{3} \mathrm{~s}^{-1}$, which differ from experimentally measured values $\left(\sim 10^{-10} \mathrm{~cm}^{3} \mathrm{~s}^{-1}\right)$ that have been corrected for photon recycling, then more exotic pathways for slowing recombination, such as some of those covered in this perspective, may indeed need strong consideration. Here we evaluated proposed mechanisms that are predicted to directly impact $k_{2}$, such as the Rashba effect and polaron formation. ${ }^{9,22,76}$ In order to better converge on the dominant recombination mechanisms controlling carrier recombination in perovskites, we explore a number of open questions related to the precise mechanisms, their relative contributions to practical devices and, importantly, how we can experimentally further scrutinize each process.

The demonstration of the PL intensity increasing as carriers thermalize to the band-edge, along with recent theoretical calculations, leads us to conclude that the Rashba effect does not significantly influence recombination in typical halide perovskite films for solar cells such as $\mathrm{MAPbI}_{3}$ and $\mathrm{MAPbBr}_{3}{ }^{52,101}$ Nevertheless, we note that the majority of high-performing $3 \mathrm{D}$ perovskite devices are no longer "simple" semiconductors made with a single halide and a single organic cation, but are now more akin to alloys containing multiple A site cations (FA, Cs, MA) 
and a mix of bromide and iodide ${ }^{116,117}$. These alloys would inherently have a lower symmetry on average because adjacent unit cells may differ. It is possible that the reduced symmetry and potential strain ${ }^{118}$ introduced by alloying the different perovskites contribute to an increased Rashba effect. In any case, devices to date are still below the radiative limits, and therefore it is likely that trapping pathways are more dominant over Rashba-related effects on $k_{2}$. Furthermore, even approaching the limits in bulk polycrystalline films which have only small deviations from perfectly symmetric crystal structures, the influence of the Rashba effect on carrier recombination would likely be negligibly smal1 ${ }^{5,101,105}$.

Polaronic effects, on the other hand, may be more pronounced, and would likely also inter-relate with other processes. It has previously been shown that polarons form more readily when charges are localized into defect states due to a reduction in the energetic barrier ${ }^{81}$ This leads to the intriguing possibility that the very act of trapping into discrete or band tail states could further inhibit recombination due to the electrostatic screening fostered by the formation of polarons. The relationship between defect density and polaron formation has not been systematically explored as most experiments probing polaron formation to date were necessarily conducted at high injected carrier densities $\left(\sim 10^{17} \mathrm{~cm}^{-3}\right)^{96}$, which are well above typical trap state densities. In addition, the rate for the diffusion-limited capture of polarons at a density of $n_{t}$ traps, $k_{1}=4 \pi D n_{t} R_{t}$, can also be lowered by polaron effects that reduce the effective capture radius $R_{\mathrm{t}}$ of traps, i.e. by introducing barriers to polaron coalescence and to deep (small-polaronic) trapping. ${ }^{71}$ Indeed, unexpectedly small values of $k_{1}$ have been reported. ${ }^{119}$ Thus, polaronic effects may work alongside trapping processes at low carrier densities $\left(<10^{16} \mathrm{~cm}^{-3}\right)$ and could explain the slow recombination rates, which has to date primarily been solely attributed to non-polaronic carriers trapping. 
This motivates future optical and electrical experiments at lower excitation fluences to better understand these processes, especially because large polarons are expected to be more stable at lower excitation powers, at which point there is less competition for nuclear polarization and therefore less interpolaron repulsion interaction. ${ }^{71,94,96}$. Passivation approaches that selectively target trapping processes ${ }^{14,50}$ may provide an ideal lever to decouple the polaronic and trapping effects. However, as trapping is reduced either through lowering defect densities or increasing carrier density, band-to-band radiative bimolecular recombination increases and photon recycling becomes increasingly important. ${ }^{115}$ Further work delineating whether recombination and trapping processes in these materials are diffusion-limited (in which polaronic contributions could explain the observed $k_{2}$ values), and/or limited by the overlap of the photoexcited electron and hole wavefunctions would help in separating some of the proposed hypothesis for reduced recombination. ${ }^{87}$

Some of these open questions may be addressed by utilizing terahertz (THz) and infrared spectroscopy as well as cyclotron resonance experiments to probe for polaron optical signatures and to measure polaron masses. The distinctively weak scattering of a large polaron causes their Drude conductivity to fall very rapidly with increasing applied frequency below characteristic phonon frequencies (e.g. microwave frequencies). ${ }^{71,120}$ In addition, excitation of a large-polaron's self-trapped electronic carrier from and within its self-trapping potential well produces a broad asymmetric absorption band above characteristic phonon frequencies. ${ }^{71,120}$ These two unusual features enable large polarons to be distinguished from conventional charge carriers, which have Drude conductivities showing very slow monotonic decreases with applied frequency through this domain. ${ }^{121}$ This unique behavior has been exploited by Munson et al., where they used timeresolved infrared spectroscopy to probe optical signatures of large polarons in $\mathrm{MAPbI}_{3} .{ }^{87}$ 
Finally, we could in principle manipulate each of these processes to generate exotic ways of controlling recombination for specific applications. If we can identify the specific source of defect screening, we could engineer materials to optimize this effect, potentially eliminating all parasitic non-radiative decay processes ${ }^{8}$. Straining perovskites through compositional engineering, growth on non-lattice matched substrates or the application of external strain could tune carrier dynamics further in these materials by artificially inducing a sizeable Rashba effect ${ }^{118,122,123}$. We could also finely tune the relativistic effects and band splitting by replacing fractions of the heavy metal atom $(\mathrm{e} . \mathrm{Pb})$ with alternatives. Recent photogalvanic measurements suggest that spin-based devices could exploit the band-splitting of the Rashba effect to achieve directional transport of excitations created with either left- or right-circularly polarized light or charge injection ${ }^{104}$; this may be useful in spin-injected field-effect transistors in which strong absorption, corresponding to a substantial indirect bandgap component, is not critical. One could also envision manipulating photon management by tweaking the composition of the perovskite to tune the absorption/emission overlap while maintaining high luminescence yields, as well as controlling light out-coupling through refractive index matching and sample geometries. ${ }^{124}$ By doing so, one could further explore efficient light extraction as well as light trapping schemes.

\section{Conclusion}

Halide perovskites are exciting materials for optoelectronic applications. In this Focus Review, we draw on the literature and on our own calculations to identify which factors impact charge carrier recombination. First, we show that multiphonon theoretical calculations derived for polar semiconductors predict long non-radiative lifetimes $\left(1 / k_{l}\right)$ for moderate trap state densities residing in the midgap region. By contrast, multiple trapping and hopping of carriers in shallow trap states 
would not lead to the observed recombination rates. Next, we evaluate the band-to-band charge carrier recombination rate constant, $k_{2}$, emphasizing that the theoretically calculated and experimentally measured internal recombination rate values are consistent with one another when corrected for photon recycling, as expected from optical reciprocity. Polaron formation has recently garnered interest through theoretical electrostatic studies as well as recent work showing optical signatures of polarons decaying more slowly at higher temperatures; further work on this topic is critical. We conclude that the Rashba effect does not meaningfully impact carrier recombination in bulk 3D structures, but may become interesting in tailored compositions or device structures which exaggerate these effects. Based on our critical assessment of the various proposed recombination pathways, we suggest future work that may allow us to better distinguish between these competing processes across different carrier densities and perovskite compositions. Understanding these processes will allow us to further exploit the unique behavior of these materials, as well as more generally generate design rules for high-performance semiconductors.

\section{SUPPORTING INFORMATION}

Derivations for simplified rate equation. Estimation of trap densities and their relationship with non-radiative lifetime. Derivation for time-resolved photoluminescence curves with and without photon recycling.

\section{AUTHOR INFORMATION}

$\underline{\text { sds65@cam.ac.uk }}$

\section{ACKNOWLEDGMENT}


D.W.D acknowledges support for this project through the MIT-Tata GridEdge Solar Research Program, which is funded by the Tata Trusts. S.D.S. acknowledges the Royal Society and Tata Group (UF150033). K.F. acknowledges a George and Lilian Schiff Studentship, Winton Studentship, the Engineering and Physical Sciences Research Council (EPSRC) studentship, Cambridge Trust Scholarship, and Robert Gardiner Scholarship. T.K. acknowledges support from the Impuls- und Vernetzungsfonds der Helmholtz Gemeinschaft via the project PEROSEED. D.S.G. acknowledges multiple sources for contributing to his understanding of carrier recombination processes in semiconductors, including his perovskite microscopy work by DOE EPSM DE-SC0013957, long-standing support fundamental semiconductor photophysics and photovoltaics studies supported by ONR N00014-17-1-2201, and recent work on defects in nanostructures by the University of Washington Molecular Engineering Materials Center (MEM-C) NSF grant DMR-1719797. The authors thank Sarah Kurtz, Jarvist Frost, Alessio Filippetti, Mark Ziffer, Laura Herz, Rohit Prasanna, Felix Deschler, Henry Snaith, and Peter Foley for valuable discussions.

\section{References}

(1) Stranks, S. D.; Petrozza, A. Revisiting photocarrier lifetimes in photovoltaics. Nat. Photonics 2016, 10, 562-562.

(2) Kurtz, S. R.; McConnell, R. Requirements for a 20\%-efficient polycrystalline GaAs solar cell. AIP Conference Proceedings 1997, 404, 191-205.

(3) Johnston, M. B.; Herz, L. M. Hybrid Perovskites for Photovoltaics: Charge-Carrier Recombination, Diffusion, and Radiative Efficiencies. Acc. Chem. Res. 2016, 49, 146154.

(4) Manser, J. S.; Kamat, P. V. Band Filling with Free Charge Carriers in Organometal Halide Perovskites. Nat. Photonics 2014, 8, 737-743.

(5) Davies, C. L.; Filip, M. R.; Patel, J. B.; Crothers, T. W.; Verdi, C.; Wright, A. D.; Milot, R. L.; Giustino, F.; Johnston, M. B.; Herz, L. M. Bimolecular recombination in methylammonium lead triiodide perovskite is an inverse absorption process. Nat.

Commun. 2018, 9, 293. 
(6) Correa-Baena, J. P.; Saliba, M.; Buonassisi, T.; Gratzel, M.; Abate, A.; Tress, W.; Hagfeldt, A. Promises and challenges of perovskite solar cells. Science 2017, 358, 739744.

(7) Richter, J. M.; Abdi-Jalebi, M.; Sadhanala, A.; Tabachnyk, M.; Rivett, J. P. H.; PazosOutón, L. M.; Gödel, K. C.; Price, M.; Deschler, F.; Friend, R. H. Enhancing photoluminescence yields in lead halide perovskites by photon recycling and light outcoupling. Nat. Commun. 2016, 7, 13941.

(8) Stranks, S. D. Nonradiative Losses in Metal Halide Perovskites. ACS Energy Lett. 2017, $2,1515-1525$.

(9) Azarhoosh, P.; McKechnie, S.; Frost, J. M.; Walsh, A.; van Schilfgaarde, M. Research Update: Relativistic origin of slow electron-hole recombination in hybrid halide perovskite solar cells. APL Materials 2016, 4, 091501.

(10) Dastidar, S.; Li, S.; Smolin, S. Y.; Baxter, J. B.; Fafarman, A. T. Slow Electron-Hole Recombination in Lead Iodide Perovskites Does Not Require a Molecular Dipole. ACS Energy Lett. 2017, 2, 2239-2244.

(11) Moser, J. E. Perovskite photovoltaics: Slow recombination unveiled. Nat. Mater. 2016, 16, 4-6.

(12) Ponseca, C. S.; Savenije, T. J.; Abdellah, M.; Zheng, K.; Yartsev, A.; Pascher, T.; Harlang, T.; Chabera, P.; Pullerits, T.; Stepanov, A.et al. Organometal Halide Perovskite Solar Cell Materials Rationalized: Ultrafast Charge Generation, High and MicrosecondLong Balanced Mobilities, and Slow Recombination. J. Am. Chem. Soc. 2014, 136, 5189 5192.

(13) Walker, A. W.; Heckelmann, S.; Karcher, C.; Höhn, O.; Went, C.; Niemeyer, M.; Bett, A. W.; Lackner, D. Nonradiative lifetime extraction using power-dependent relative photoluminescence of III-V semiconductor double-heterostructures. J. Appl. Phys. 2016, $119,155702$.

(14) deQuilettes, D. W.; Koch, S.; Burke, S.; Paranji, R. K.; Shropshire, A. J.; Ziffer, M. E.; Ginger, D. S. Photoluminescence Lifetimes Exceeding $8 \mu$ s and Quantum Yields Exceeding $30 \%$ in Hybrid Perovskite Thin Films by Ligand Passivation. ACS Energy Lett. 2016, 438-444.

(15) Brenes, R.; Guo, D.; Osherov, A.; Noel, N. K.; Eames, C.; Hutter, E. M.; Pathak, S. K.; Niroui, F.; Friend, R. H.; Islam, M. S.et al. Metal Halide Perovskite Polycrystalline Films Exhibiting Properties of Single Crystals. Joule 2017, 1, 155-167.

(16) Green, M. A.; Ho-Baillie, A.; Snaith, H. J. The emergence of perovskite solar cells. Nat. Photonics 2014, 8, 506-514.

(17) Kirchartz, T.; Rau, U. Decreasing Radiative Recombination Coefficients via an Indirect Band Gap in Lead Halide Perovskites. J. Phys. Chem. Lett. 2017, 8, 1265-1271.

(18) van Roosbroeck, W.; Shockley, W. Photon-Radiative Recombination of Electrons and Holes in Germanium. Phys. Rev. 1954, 94, 1558-1560.

(19) Cardona, P. Y. a. M. Fundamentals of Semiconductors: Physics and Materials Properties. Springer-Verlag Berlin Heidelberg 2010, 4.

(20) Staub, F.; Hempel, H.; Hebig, J.-C.; Mock, J.; Paetzold, U. W.; Rau, U.; Unold, T.; Kirchartz, T. Beyond Bulk Lifetimes: Insights into Lead Halide Perovskite Films from Time-Resolved Photoluminescence. Phys. Rev. Appl. 2016, 6, 044017. 
(21) Filippetti, A.; Delugas, P.; Mattoni, A. Radiative Recombination and Photoconversion of Methylammonium Lead Iodide Perovskite by First Principles: Properties of an Inorganic Semiconductor within a Hybrid Body. J. Phys. Chem. C 2014, 118, 24843-24853.

(22) Chen, Y.; Yi, H. T.; Wu, X.; Haroldson, R.; Gartstein, Y. N.; Rodionov, Y. I.; Tikhonov, K. S.; Zakhidov, A.; Zhu, X. Y.; Podzorov, V. Extended carrier lifetimes and diffusion in hybrid perovskites revealed by Hall effect and photoconductivity measurements. Nat. Commun. 2016, 7, 12253.

(23) Saba, M.; Cadelano, M.; Marongiu, D.; Chen, F.; Sarritzu, V.; Sestu, N.; Figus, C.; Aresti, M.; Piras, R.; Lehmann, A. G.et al. Correlated Electron-Hole Plasma in Organometal Perovskites. Nat. Commun. 2014, 5, 5049.

(24) Crothers, T. W.; Milot, R. L.; Patel, J. B.; Parrott, E. S.; Schlipf, J.; Müller-Buschbaum, P.; Johnston, M. B.; Herz, L. M. Photon Reabsorption Masks Intrinsic Bimolecular Charge-Carrier Recombination in CH3NH3PbI3 Perovskite. Nano Lett. 2017, 17, 57825789.

(25) Richter, J. M.; Abdi-Jalebi, M.; Sadhanala, A.; Tabachnyk, M.; Rivett, J. P.; PazosOuton, L. M.; Godel, K. C.; Price, M.; Deschler, F.; Friend, R. H. Enhancing Photoluminescence Yields in Lead Halide Perovskites by Photon Recycling and Light Out-Coupling. Nat. Commun. 2016, 7, 13941.

(26) Walsh, A.; Zunger, A. Instilling defect tolerance in new compounds. Nat. Mater. 2017, 16, 964.

(27) Ball, J. M.; Petrozza, A. Defects in Perovskite-Halides and Their Effects in Solar Cells. Nat. Energy 2016, 1, 16149.

(28) Huang, J.; Yuan, Y.; Shao, Y.; Yan, Y. Understanding the physical properties of hybrid perovskites for photovoltaic applications. Nat. Rev. Mater. 2017, 2, 17042.

(29) deQuilettes, D. W.; Vorpahl, S. M.; Stranks, S. D.; Nagaoka, H.; Eperon, G. E.; Ziffer, M. E.; Snaith, H. J.; Ginger, D. S. Impact of Microstructure on Local Carrier Lifetime in Perovskite Solar Cells. Science 2015, 348, 683-686.

(30) Herz, L. M. Charge-Carrier Dynamics in Organic-Inorganic Metal Halide Perovskites. Annu. Rev. Phys. Chem. 2016, 67, 65-89.

(31) Manser, J. S.; Christians, J. A.; Kamat, P. V. Intriguing Optoelectronic Properties of Metal Halide Perovskites. Chemical Reviews 2016, 116, 12956-13008.

(32) Wright, A. D.; Milot, R. L.; Eperon, G. E.; Snaith, H. J.; Johnston, M. B.; Herz, L. M. Band-Tail Recombination in Hybrid Lead Iodide Perovskite. Adv. Funct. Mater. 2017, 27, 1700860 .

(33) Leijtens, T.; Eperon, G. E.; Barker, A. J.; Grancini, G.; Zhang, W.; Ball, J. M.; Kandada, A. R. S.; Snaith, H. J.; Petrozza, A. Carrier trapping and recombination: the role of defect physics in enhancing the open circuit voltage of metal halide perovskite solar cells. Energy Environ. Sci. 2016, 9, 3472-3481.

(34) Wu, X.; Trinh, M. T.; Niesner, D.; Zhu, H.; Norman, Z.; Owen, J. S.; Yaffe, O.; Kudisch, B. J.; Zhu, X. Y. Trap states in lead iodide perovskites. J. Am. Chem. Soc. 2015, 137, 2089-2096.

(35) Adinolfi, V.; Yuan, M.; Comin, R.; Thibau, E. S.; Shi, D.; Saidaminov, M. I.; Kanjanaboos, P.; Kopilovic, D.; Hoogland, S.; Lu, Z.-H.et al. The In-Gap Electronic State Spectrum of Methylammonium Lead Iodide Single-Crystal Perovskites. Adv. Mater. 2016, 28, 3406-3410. 
(36) Henry, C. H.; Lang, D. V. Nonradiative capture and recombination by multiphonon emission in GaAs and GaP. Phys. Rev. B 1977, 15, 989-1016.

(37) Ridley, B. K. On the multiphonon capture rate in semiconductors. Solid-State Electronics 1978, 21, 1319-1323.

(38) Ridley, B. K. Quantum Processes in Semiconductors. Oxford University Press 2013, Oxford, U.K.

(39) Markvart, T. Multiphonon recombination. In Recombination in Semiconductors. Landsberg, P.T., Ed.; Cambridge University Press: Cambride, U.K. 2003, 467-468.

(40) Monahan, D. M.; Guo, L.; Lin, J.; Dou, L.; Yang, P.; Fleming, G. R. Room-Temperature Coherent Optical Phonon in 2D Electronic Spectra of CH3NH3PbI3 Perovskite as a Possible Cooling Bottleneck. J. Phys. Chem. Lett. 2017, 8, 3211-3215.

(41) Kirchartz, T.; Markvart, T.; Rau, U.; Egger, D. A. Impact of Small Phonon Energies on the Charge-Carrier Lifetimes in Metal-Halide Perovskites. J. Phys. Chem. Lett. 2018, 9, 939-946.

(42) Sendner, M.; Nayak, P. K.; Egger, D. A.; Beck, S.; Muller, C.; Epding, B.; Kowalsky, W.; Kronik, L.; Snaith, H. J.; Pucci, A.et al. Optical phonons in methylammonium lead halide perovskites and implications for charge transport. Mater. Horiz. 2016, 3, 613-620.

(43) Pankove, J. I. Optical Processes in Semiconductors. Prentice-Hall, Inc. 1971, Englewood Cliffs, N.J.

(44) Johnson, S. R.; Ding, D.; Wang, J. B.; Yu, S. Q.; Zhang, Y. H. Excitation dependent photoluminescence measurements of the nonradiative lifetime and quantum efficiency in GaAs. J. Vac. Sci. Technol. B 2007, 25, 1077-1082.

(45) Riley E. Brandt, V. S., David S. Ginley, Tonio Buonassisi. Identifying Defect-Tolerant Semiconductors with High Minority-Carrier Lifetimes: Beyond Hybrid Lead Halide Perovskites. MRS Commun. 2015, 5, 265-275.

(46) Chirvony, V. S.; Martínez-Pastor, J. P. Trap-Limited Dynamics of Excited Carriers and Interpretation of the Photoluminescence Decay Kinetics in Metal Halide Perovskites. $J$. Phys. Chem. Lett. 2018, 9, 4955-4962.

(47) Yaffe, O.; Guo, Y.; Tan, L. Z.; Egger, D. A.; Hull, T.; Stoumpos, C. C.; Zheng, F.; Heinz, T. F.; Kronik, L.; Kanatzidis, M. G.et al. Local Polar Fluctuations in Lead Halide Perovskite Crystals. Phys. Rev. Lett. 2017, 118, 136001.

(48) Frost, J. M.; Walsh, A. What Is Moving in Hybrid Halide Perovskite Solar Cells? Accounts Chem. Res. 2016, 49, 528-535.

(49) Noel, N. K.; Abate, A.; Stranks, S. D.; Parrott, E. S.; Burlakov, V. M.; Goriely, A.; Snaith, H. J. Enhanced Photoluminescence and Solar Cell Performance via Lewis Base Passivation of Organic-Inorganic Lead Halide Perovskites. ACS Nano 2014, 8, 9815 9821.

(50) Abdi-Jalebi, M.; Andaji-Garmaroudi, Z.; Cacovich, S.; Stavrakas, C.; Philippe, B.; Richter, J. M.; Alsari, M.; Booker, E. P.; Hutter, E. M.; Pearson, A. J.et al. Maximizing and stabilizing luminescence from halide perovskites with potassium passivation. Nature 2018, 555, 497-501.

(51) Milot, R. L.; Eperon, G. E.; Snaith, H. J.; Johnston, M. B.; Herz, L. M. TemperatureDependent Charge-Carrier Dynamics in $\mathrm{CH}_{3} \mathrm{NH}_{3} \mathrm{PbI}_{3}$ Perovskite Thin Films. Adv. Funct. Mater. 2015, 25, 6218-6227. 
(52) Richter, J. M.; Chen, K.; Sadhanala, A.; Butkus, J.; Rivett, J. P. H.; Friend, R. H.; Monserrat, B.; Hodgkiss, J. M.; Deschler, F. Direct Bandgap Behavior in Rashba-Type Metal Halide Perovskites. Adv. Mater. 2018, 30, 1803379.

(53) Staub, F.; Rau, U.; Kirchartz, T. Statistics of the Auger Recombination of Electrons and Holes via Defect Levels in the Band Gap-Application to Lead-Halide Perovskites. ACS Omega 2018, 3, 8009-8016.

(54) Draguta, S.; Thakur, S.; Morozov, Y. V.; Wang, Y.; Manser, J. S.; Kamat, P. V.; Kuno, M. Spatially Non-uniform Trap State Densities in Solution-Processed Hybrid Perovskite Thin Films. J. Phys. Chem. Lett. 2016, 7, 715-721.

(55) Merdasa, A.; Kiligaridis, A.; Rehermann, C.; Abdi-Jalebi, M.; Stöber, J.; Louis, B.; Gerhard, M.; Stranks, S. D.; Unger, E. L.; Scheblykin, I. G. Impact of Excess Lead Iodide on the Recombination Kinetics in Metal Halide Perovskites. ACS Energy Lett. 2019, 4, 1370-1378.

(56) Tennyson, E. M.; Doherty, T. A. S.; Stranks, S. D. Heterogeneity at multiple length scales in halide perovskite semiconductors. Nat. Rev. Mater. 2019.

(57) deQuilettes, D. W.; Jariwala, S.; Burke, S.; Ziffer, M. E.; Wang, J. T. W.; Snaith, H. J.; Ginger, D. S. Tracking Photoexcited Carriers in Hybrid Perovskite Semiconductors: Trap-Dominated Spatial Heterogeneity and Diffusion. ACS Nano 2017, 11, 11488-11496.

(58) Grancini, G.; Viola, D.; Gandini, M.; Altamura, D.; Pogna, E. A. A.; D’Innocenzo, V.; Bargigia, I.; Giannini, C.; Cerullo, G.; Petrozza, A. Lattice Distortions Drive ElectronHole Correlation within Micrometer-Size Lead-Iodide Perovskite Crystals. ACS Energy Lett. 2017, 2, 265-269.

(59) Birkhold, S. T.; Precht, J. T.; Liu, H.; Giridharagopal, R.; Eperon, G. E.; Schmidt-Mende, L.; Li, X.; Ginger, D. S. Interplay of Mobile Ions and Injected Carriers Creates Recombination Centers in Metal Halide Perovskites under Bias. ACS Energy Lett. 2018, 3, 1279-1286.

(60) Meggiolaro, D.; De Angelis, F. First-Principles Modeling of Defects in Lead Halide Perovskites: Best Practices and Open Issues. ACS Energy Lett. 2018, 3, 2206-2222.

(61) Bhattacharya, R.; Pal, B.; Bansal, B. On conversion of luminescence into absorption and the van Roosbroeck-Shockley relation. App. Phys. Lett. 2012, 100, 222103.

(62) Varshni, Y. P. Band-to-Band Radiative Recombination in Groups IV, VI, and III-V Semiconductors (I). phys. stat. sol. (b) 1967, 19, 459-514.

(63) Staub, F.; Kirchartz, T.; Bittkau, K.; Rau, U. Manipulating the Net Radiative Recombination Rate in Lead Halide Perovskite Films by Modification of Light Outcoupling. J. Phys. Chem. Lett. 2017, 8, 5084-5090.

(64) Trupke, T.; Green, M. A.; Würfel, P.; Altermatt, P. P.; Wang, A.; Zhao, J.; Corkish, R. Temperature dependence of the radiative recombination coefficient of intrinsic crystalline silicon. J. Appl. Phys. 2003, 94, 4930-4937.

(65) Stern, F. Gain-current relation for GaAs lasers with n-type and undoped active layers. IEEE J. Quantum Elect. 1973, 9, 290-294.

(66) Muth, J. F.; Lee, J. H.; Shmagin, I. K.; Kolbas, R. M.; Casey, H. C.; Keller, B. P.; Mishra, U. K.; DenBaars, S. P. Absorption coefficient, energy gap, exciton binding energy, and recombination lifetime of $\mathrm{GaN}$ obtained from transmission measurements. Appl. Phys. Lett. 1997, 71, 2572-2574.

(67) Lush, G. B. B-coefficient in n-type GaAs. Sol. Energ. Mat. Sol. C. 2009, 93, 1225-1229. 
(68) Ahrenkiel, R. K.; Keyes, B. M.; Lush, G. B.; Melloch, M. R.; Lundstrom, M. S.; MacMillan, H. F. Minority- carrier lifetime and photon recycling in n- GaAs. J. Vac. Sci. Technol. A 1992, 10, 990-995.

(69) Nelson, R. J.; Sobers, R. G. Minority- carrier lifetimes and internal quantum efficiency of surface- free GaAs. J. Appl. Phys. 1978, 49, 6103-6108.

(70) Zhang, X.; Shen, J.-X.; Wang, W.; Van de Walle, C. G. First-Principles Analysis of Radiative Recombination in Lead-Halide Perovskites. ACS Energy Lett. 2018, 3, 23292334.

(71) Emin, D. Polarons. Cambridge University Press, New York 2013.

(72) Emin, D. On the existence of free and self-trapped carriers in insulators: an abrupt temperature-dependent conductivity transition. Advances in Physics 1973, 22, 57-116.

(73) Whitfield, C. G. K. a. G. D. Polarons and Excitons. Plenum Press, New York 1963.

(74) Landau, L. D. On the motion of electrons in a crystal lattice. Phys. Z. Sowjetunion 1933, 3, 664-665.

(75) Bonn, M.; Miyata, K.; Hendry, E.; Zhu, X. Y. Role of Dielectric Drag in Polaron Mobility in Lead Halide Perovskites. ACS Energy Lett. 2017, 2, 2555-2562.

(76) Podzorov, X.-Y. Z. a. V. Charge Carriers in Hybrid Organic-Inorganic Lead Halid Perovskites Might Be Protected as Large Polaron. J. Phys. Chem. Lett. 2015, 6, 4.

(77) Henry Ehrenreich, F. S., David Turnbull. Solid State Physics. Academic Press 1980, 35 , Tables I and IV.

(78) Juarez-Perez, E. J.; Sanchez, R. S.; Badia, L.; Garcia-Belmonte, G.; Kang, Y. S.; MoraSero, I.; Bisquert, J. Photoinduced Giant Dielectric Constant in Lead Halide Perovskite Solar Cells. J. Phys. Chem. Lett. 2014, 5, 2390-2394.

(79) Guerrero, A.; Garcia-Belmonte, G.; Mora-Sero, I.; Bisquert, J.; Kang, Y. S.; Jacobsson, T. J.; Correa-Baena, J.-P.; Hagfeldt, A. Properties of Contact and Bulk Impedances in Hybrid Lead Halide Perovskite Solar Cells Including Inductive Loop Elements. J. Phys. Chem. C 2016, 120, 8023-8032.

(80) Herz, L. M. How Lattice Dynamics Moderate the Electronic Properties of Metal-Halide Perovskites. J. Phys. Chem. Lett. 2018, 9, 6853-6863.

(81) Emin, D.; Holstein, T. Adiabatic Theory of an Electron in a Deformable Continuum. Phys. Rev. Lett. 1976, 36, 323-326.

(82) Neukirch, A. J.; Nie, W.; Blancon, J.-C.; Appavoo, K.; Tsai, H.; Sfeir, M. Y.; Katan, C.; Pedesseau, L.; Even, J.; Crochet, J. J.et al. Polaron Stabilization by Cooperative Lattice Distortion and Cation Rotations in Hybrid Perovskite Materials. Nano Lett. 2016, 16, 3809-3816.

(83) Herz, L. M. Charge-Carrier Mobilities in Metal Halide Perovskites: Fundamental Mechanisms and Limits. ACS Energy Lett. 2017, 2, 1539-1548.

(84) Emin, D. Small Polarons. Phys. Today 1982, 35, 34-40.

(85) Emin, D. Barrier to recombination of oppositely charged large polarons. J. Appl. Phys. 2018, 123, 055105.

(86) Wehrenfennig, C.; Eperon, G. E.; Johnston, M. B.; Snaith, H. J.; Herz, L. M. High Charge Carrier Mobilities and Lifetimes in Organolead Trihalide Perovskites. $A d v$. Mater. 2014, 26, 1584-1589.

(87) Munson, K. T.; Kennehan, E. R.; Doucette, G. S.; Asbury, J. B. Dynamic Disorder Dominates Delocalization, Transport, and Recombination in Halide Perovskites. Chem 2018, 4, 2826-2843. 
(88) Schooley, J. F.; Hosler, W. R.; Cohen, M. L. Superconductivity in Semiconducting SrTiO3. Phys. Rev. Lett. 1964, 12, 474-475.

(89) Bednorz, J. G.; Müller, K. A. Possible high Tc superconductivity in the $\mathrm{Ba}-\mathrm{La}-\mathrm{Cu}-\mathrm{O}$ system. Z. Phys. B Con. Mat. 1986, 64, 189-193.

(90) Emin, D. In-plane conductivity of a layered large-bipolaron liquid. Philos. Mag. 2015, 95, 918-934.

(91) Emin, D. Formation, motion, and high-temperature superconductivity of large bipolarons. Phys. Rev. Lett. 1989, 62, 1544-1547.

(92) Emin, D. Proposed high-power beta cells from MgAlB14-type icosahedral-boron semiconductors. AIP Adv. 2019, 9, 055226.

(93) Miyata, K.; Meggiolaro, D.; Trinh, M. T.; Joshi, P. P.; Mosconi, E.; Jones, S. C.; De Angelis, F.; Zhu, X. Y. Large polarons in lead halide perovskites. Sci. Adv. 2017, 3, e1701217.

(94) Miyata, K.; Atallah, T. L.; Zhu, X. Y. Lead halide perovskites: Crystal-liquid duality, phonon glass electron crystals, and large polaron formation. Sci. Adv. 2017, 3, e1701469.

(95) Zhu, H.; Trinh, M. T.; Wang, J.; Fu, Y.; Joshi, P. P.; Miyata, K.; Jin, S.; Zhu, X. Y. Organic Cations Might Not Be Essential to the Remarkable Properties of Band Edge Carriers in Lead Halide Perovskites. Adv. Mater. 2017, 29, 1603072.

(96) Zhu, H.; Miyata, K.; Fu, Y.; Wang, J.; Joshi, P. P.; Niesner, D.; Williams, K. W.; Jin, S.; Zhu, X. Y. Screening in crystalline liquids protects energetic carriers in hybrid perovskites. Science 2016, 353, 1409-1413.

(97) Niesner, D.; Zhu, H.; Miyata, K.; Joshi, P. P.; Evans, T. J. S.; Kudisch, B. J.; Trinh, M. T.; Marks, M.; Zhu, X. Y. Persistent Energetic Electrons in Methylammonium Lead Iodide Perovskite Thin Films. J. Am. Chem. Soc. 2016, 138, 15717-15726.

(98) Gong, J.; Yang, M.; Ma, X.; Schaller, R. D.; Liu, G.; Kong, L.; Yang, Y.; Beard, M. C.; Lesslie, M.; Dai, Y.et al. Electron-Rotor Interaction in Organic-Inorganic Lead Iodide Perovskites Discovered by Isotope Effects. J. Phys. Chem. Lett. 2016, 7, 2879-2887.

(99) Zheng, F.; Tan, L. Z.; Liu, S.; Rappe, A. M. Rashba Spin-Orbit Coupling Enhanced Carrier Lifetime in $\mathrm{CH}_{3} \mathrm{NH}_{3} \mathrm{PbI}_{3}$. Nano Lett. 2015, 15, 7794-7800.

(100) Bychkov, Y. A.; Rashba, E. I. Properties of a 2D electron gas with lifted spectral degeneracy. J. Exp. Theor. Phys. Lett. 1984, 39, 78-81.

(101) Frohna, K.; Deshpande, T.; Harter, J.; Peng, W.; Barker, B. A.; Neaton, J. B.; Louie, S. G.; Bakr, O.; Hsieh, D.; Bernardi, M. Inversion Symmetry and Bulk Rashba Effect in Methylammonium Lead Iodide Perovskite Single Crystals. Nat. Commun. 2018, 9, 1829.

(102) Niesner, D.; Wilhelm, M.; Levchuk, I.; Osvet, A.; Shrestha, S.; Batentschuk, M.; Brabec, C.; Fauster, T. Giant Rashba Splitting in $\mathrm{CH}_{3} \mathrm{NH}_{3} \mathrm{PbBr}_{3}$ Organic-Inorganic Perovskite. Phys. Rev. Lett. 2016, 117, 126401.

(103) Isarov, M.; Tan, L. Z.; Bodnarchuk, M. I.; Kovalenko, M. V.; Rappe, A. M.; Lifshitz, E. Rashba Effect in a Single Colloidal CsPbBr3 Perovskite Nanocrystal Detected by Magneto-Optical Measurements. Nano Lett. 2017, 17, 5020-5026.

(104) Niesner, D.; Hauck, M.; Shrestha, S.; Levchuk, I.; Matt, G. J.; Osvet, A.; Batentschuk, M.; Brabec, C.; Weber, H. B.; Fauster, T. Structural fluctuations cause spin-split states in tetragonal (CH3NH3)PbI3 as evidenced by the circular photogalvanic effect. P. Natl. Acad. Sci. USA 2018, 115, 9509-9514.

(105) Stranks, S. D.; Plochocka, P. The influence of the Rashba effect. Nat. Mater. 2018, 17, 381-382. 
(106) Hutter, E. M.; Savenije, T. J. Thermally Activated Second-Order Recombination Hints toward Indirect Recombination in Fully Inorganic CsPbI3 Perovskites. ACS Energy Lett. 2018, 3, 2068-2069.

(107) Sarritzu, V.; Sestu, N.; Marongiu, D.; Chang, X.; Wang, Q.; Masi, S.; Colella, S.; Rizzo, A.; Gocalinska, A.; Pelucchi, E.et al. Direct or Indirect Bandgap in Hybrid Lead Halide Perovskites? Adv. Opt. Mater. 2018, 6, 1701254.

(108) Pazos-Outon, L. M.; Szumilo, M.; Lamboll, R.; Richter, J. M.; Crespo-Quesada, M.; Abdi-Jalebi, M.; Beeson, H. J.; Vrucinic, M.; Alsari, M.; Snaith, H. J.et al. Photon Recycling in Lead Iodide Perovskite Solar Cells. Science 2016, 351, 1430-1433.

(109) Kirchartz, T.; Staub, F.; Rau, U. Impact of Photon Recycling on the Open-Circuit Voltage of Metal Halide Perovskite Solar Cells. ACS Energy Lett. 2016, 1, 731-739.

(110) Martí, A.; Balenzategui, J. L.; Reyna, R. F. Photon recycling and Shockley's diode equation. J. Appl. Phys. 1997, 82, 4067-4075.

(111) Asbeck, P. Self- absorption effects on the radiative lifetime in GaAs- GaAlAs double heterostructures. J. Appl. Phys. 1977, 48, 820-822.

(112) Ahrenkiel, R. K.; Dunlavy, D. J.; Keyes, B.; Vernon, S. M.; Dixon, T. M.; Tobin, S. P.; Miller, K. L.; Hayes, R. E. Ultralong minority- carrier lifetime epitaxial GaAs by photon recycling. Appl. Phys. Lett. 1989, 55, 1088-1090.

(113) Braly, I. L.; deQuilettes, D. W.; Pazos-Outón, L. M.; Burke, S.; Ziffer, M. E.; Ginger, D. S.; Hillhouse, H. W. Hybrid perovskite films approaching the radiative limit with over 90\% photoluminescence quantum efficiency. Nat. Photonics 2018, 12, 355-361.

(114) Yablonovitch, E.; Miller, O. D.; Kurtz, S. R. 2012 38th IEEE Photovoltaic Specialists Conference, 2012; p 001556-001559.

(115) Brenes, R.; Laitz, M.; Jean, J.; deQuilettes, D. W.; Bulović, V. Benefit from Photon Recycling at the Maximum-Power Point of State-of-the-Art Perovskite Solar Cells. Phys. Rev. Appl. 2019, 12, 014017.

(116) Yang, W. S.; Park, B. W.; Jung, E. H.; Jeon, N. J.; Kim, Y. C.; Lee, D. U.; Shin, S. S.; Seo, J.; Kim, E. K.; Noh, J. H.et al. Iodide management in formamidinium-lead-halidebased perovskite layers for efficient solar cells. Science 2017, 356, 1376-1379.

(117) Saliba, M.; Matsui, T.; Domanski, K.; Seo, J. Y.; Ummadisingu, A.; Zakeeruddin, S. M.; Correa-Baena, J. P.; Tress, W. R.; Abate, A.; Hagfeldt, A.et al. Incorporation of rubidium cations into perovskite solar cells improves photovoltaic performance. Science 2016, 354, 206-209.

(118) Leppert, L.; Reyes-Lillo, S. E.; Neaton, J. B. Electric Field- and Strain-Induced Rashba Effect in Hybrid Halide Perovskites. J. Phys. Chem. Lett. 2016, 7, 3683-3689.

(119) Wiktor, J.; Ambrosio, F.; Pasquarello, A. Mechanism suppressing charge recombination at iodine defects in $\mathrm{CH} 3 \mathrm{NH} 3 \mathrm{PbI} 3$ by polaron formation. J. Mater. Chem. A 2018, 6, 16863-16867.

(120) Emin, D. Optical properties of large and small polarons and bipolarons. Phys. Rev. B 1993, 48, 13691-13702.

(121) Emin, D. Dynamic d-symmetry Bose condensate of a planar-large-bipolaron liquid in cuprate superconductors. Philos. Mag. 2017, 97, 2931-2945.

(122) Odenthal, P.; Talmadge, W.; Gundlach, N.; Wang, R.; Zhang, C.; Sun, D.; Yu, Z.-G.; Valy Vardeny, Z.; Li, Y. S. Spin-polarized exciton quantum beating in hybrid organicinorganic perovskites. Nat. Phys. 2017, 13, 894. 
(123) Jones, T. W.; Osherov, A.; Alsari, M.; Sponseller, M.; Duck, B. C.; Jung, Y.-K.; Settens, C.; Niroui, F.; Brenes, R.; Stan, C. V.et al. Lattice strain causes non-radiative losses in halide perovskites. Energy Environ. Sci. 2019, 12, 596-606.

(124) Stranks, S. D.; Hoye, R. L. Z.; Di, D.; Friend, R. H.; Deschler, F. The Physics of Light Emission in Halide Perovskite Devices. Adv. Mater. 2018, 0, 1803336. 